\title{
Radiolucent lesions of the mandible: a pattern-based approach to diagnosis
}

\author{
Laurène Avril • Tommaso Lombardi • Angeliki Ailianou • \\ Karim Burkhardt • Arthur Varoquaux • Paolo Scolozzi • \\ Minerva Becker
}

Received: 20 September 2013 / Revised: 1 November 2013 / Accepted: 6 November 2013 / Published online: 10 December 2013

(C) The Author(s) 2013. This article is published with open access at Springerlink.com

\begin{abstract}
Objectives Radiolucent mandibular lesions seen on panoramic radiographs develop from both odontogenic and nonodontogenic structures. They represent a broad spectrum of lesions with a varying degree of malignant potential. The purpose of this review is to illustrate the characteristic imaging findings - as well as the clinical and histological features - of common and uncommon radiolucent lesions of the mandible. Methods This review article is based on the retrospective evaluation of 11,725 panoramic radiographs seen in our institution during the past 6 years. It provides a comprehensive, practical approach to the radiological interpretation of radiolucent lesions of the mandible. To facilitate the diagnostic approach, we have classified radiolucent lesions into two groups: lesions with well-defined borders and those with illdefined borders.

Results Lesion prevalence, age of manifestation, location within the mandible, relationship to dental structures, effect on adjacent structures and characteristic findings at computed tomography $(\mathrm{CT})$, cone beam $\mathrm{CT}(\mathrm{CBCT})$ and magnetic resonance imaging (MRI) with diffusion-weighted imaging
\end{abstract}

L. Avril $\cdot$ A. Ailianou $\cdot$ A. Varoquaux $\cdot$ M. Becker $(\bowtie)$

Department of Radiology, University Hospital of Geneva,

University of Geneva, Rue Gabrielle-Perret-Gentil 4,

1211 Geneva 14, Switzerland

e-mail: Minerva.Becker@hcuge.ch

T. Lombardi $\cdot$ P. Scolozzi

Department of Maxillo-Facial Surgery, University Hospital of

Geneva, University of Geneva, Rue Gabrielle-Perret-Gentil 4,

1211 Geneva 14, Switzerland

K. Burkhardt

Department of Clinical Pathology, University Hospital of Geneva,

University of Geneva, Rue Gabrielle-Perret-Gentil 4,

1211 Geneva 14, Switzerland
(DWI) are discussed. Pitfalls including malignant lesions mimicking benign disease and pseudo-lesions are equally addressed.

Conclusion Knowledge of the characteristic imaging features of radiolucent mandibular lesions narrows the differential diagnosis and is crucial for the identification of those lesions, where biopsy is indicated for definitive histology.

Teaching points

- Panoramic X-rays, CT and MRI are essential for the workup of radiolucent mandibular lesions.

- Lesion borders, location within the mandible, relationship to dental structures and tissue characteristics on crosssectional imaging are indispensable to narrow the differential diagnosis.

- High-resolution CT and CBCT play a major role for the assessment of lesion margins and their relationship to important anatomic structures, such as the inferior alveolar nerve.

- Although most radiolucent lesions with well-defined sclerotic borders are benign, MRI may reveal clinically unsuspected malignant disease.

Keywords Mandible $\cdot$ Radiolucent lesions $\cdot$ Panoramic radiography $\cdot$ Computed tomography $(\mathrm{CT}) \cdot$ Magnetic resonance imaging (MRI)

$\begin{array}{ll}\text { Abbreviations } \\ \text { ADC } & \text { Apparent diffusion coefficient } \\ \text { AAOMS } & \begin{array}{c}\text { American Association of Oral and Maxillofacial } \\ \text { Surgeons }\end{array} \\ \text { BRONJ } & \text { Bisphosphonate-related osteonecrosis of the jaw } \\ \text { CBCT } & \text { Cone beam computed tomography } \\ \text { CT } & \text { Computed tomography } \\ \text { DWI } & \text { Diffusion-weighted imaging } \\ \text { EG } & \text { Eospinophilic granuloma } \\ \text { GCG } & \text { Giant cell granuloma }\end{array}$




$\begin{array}{ll}\text { HL } & \text { Hodgkin lymphoma } \\ \text { KCOT } & \text { Keratocystic odontogenic tumour } \\ \text { LCH } & \text { Langerhans cell histiocytosis } \\ \text { MRI } & \text { Magnetic resonance imaging } \\ \text { NHL } & \text { Non-Hodgkin lymphoma } \\ \text { OPT } & \text { Orthopantomography } \\ \text { ORN } & \text { Osteoradionecrosis } \\ \text { PET/CT } & \text { Positron emission tomography combined } \\ & \text { with CT } \\ \text { PET/MRI } & \begin{array}{l}\text { Positron emission tomography combined } \\ \end{array} \\ \text { SBith MRI } \\ \text { SCC } & \text { Simple bone cyst } \\ \text { SUV } & \text { Squamous cell carcinoma } \\ & \text { Standardised uptake value }\end{array}$

\section{Introduction}

Conventional radiography may reveal a variety of radiolucent lesions in the mandible. These represent a broad spectrum of odontogenic and non-odontogenic lesions with a varying degree of malignant potential. Interpretation of radiolucent lesions of the mandible can be challenging either because the clinical presentation may be non-specific or because the lesion may be detected incidentally $[1,2]$. In some cases, the diagnosis will be mainly made based on clinical symptoms. In other cases, although a thorough clinical evaluation is mandatory, clinical findings are non-contributory, as the lesion cannot be seen or palpated and laboratory findings are not abnormal. Imaging is essential not only for the diagnosis of mandibular lesions but also to guide therapy and to monitor treatment response. Although common, mandibular lesions are not frequently imaged by radiologists. Nevertheless, their recognition is essential for a rapid and correct diagnosis. This review provides a comprehensive, practical approach to the radiological interpretation of radiolucent lesions of the mandible and discusses their clinical presentation and pathophysiology. It focuses on the radiological techniques used and their respective role for the assessment of these lesions.

\section{Imaging modalities}

Due to the easy access and low radiation dose, conventional radiographs such as panoramic radiographs (orthopantomographies [OPTs] or panoramix X-rays) as well as dental intraoral radiographs traditionally form the backbone in the diagnosis of osseous changes in the mandible $[1,3]$. In addition, the introduction of digital radiographs has lead to further dose reduction (up to $80 \%$ ) with the possibility of densitometric and subtraction techniques [4]. Conventional radiographs of the mandible, typically OPTs, may reveal radiolucent, radiodense or mixed pattern lesions $[1,3]$. In many cases, such as in radicular cysts, the diagnosis is straightforward and no additional imaging is required for diagnosis and treatment. As conventional radiographs are two-dimensional projections of three-dimensional structures, they have a limited value for the assessment of lesion size, lesion margins, as well as extension into important anatomic structures or soft tissues. Computed tomography (CT), cone beam CT $(\mathrm{CBCT})$, magnetic resonance imaging (MRI) and positron emission tomography combined with CT (PET/CT) and more recently positron emission tomography combined with MRI (PET/MRI) complement conventional radiographs overcoming the above-mentioned limitations and providing more specific information in terms of diagnosis and therapeutic options.

Thin-slice (1-mm), high-resolution CT with bone window settings is mainly used pre-operatively to precisely assess lesion size, margins, destruction and expansion patterns, as well as the relationship of the lesion to the mandibular canal. Although coronal slices are sufficient in many situations, dental CT with orthoradial and panoramic reconstructions are superior to standard coronal reconstructions for the evaluation of the relationship of a lesion to the dental structures and to the mandibular canal. Intravenous contrast material is mainly used in cases of suspected jaw infection or in neoplastic diseases to assess the intraosseous and extraosseous involvement. Although CBCT has gained increasing popularity over the past years, it does not allow evaluation of extraosseous structures; use of CBCT may therefore lead to underestimation of disease extent.

High-resolution MRI is mainly used as a complementary tool to CT or CBCT, as it allows precise depiction of intraosseous and extraosseous lesion components, cyst wall architecture (thin versus irregular walls, mural nodules, papillary projections), enhancement patterns after intravenous administration of gadolinium chelates (mild to strong), and type of soft tissue involvement (displacement versus infiltration) [5]. In inflammatory and infectious lesions, MRI is more sensitive than CT or CBCT for the detection of bone marrow involvement [6].

Diffusion weighted imaging (DWI) is a functional MRI technique based on the assessment of random (Brownian) motion of water molecules. Biological barriers can impair the free displacement of water molecules, thus resulting in restricted diffusivity. Restricted diffusivity is seen in a variety of conditions, including stroke, tumours with increased cellularity, infection and inflammation, as well as abscesses. Diffusion in biological tissues can be quantified using the apparent diffusion coefficient (ADC). ADC measurements (in $\mathrm{mm}^{2} / \mathrm{s}$ ) have been shown to be reproducible with excellent intraobserver and interobserver reproducibility in the head and neck [7]. MRI with DWI and ADC measurements helps in the differential diagnosis of cysts, ameloblastomas and malignant tumours (see below). Although ADC values cannot predict the histological grade in head and neck squamous cell carcinoma 
(SCC), lower values are observed in poorly differentiated lesions whereas higher values are seen in well-differentiated tumours [7]. In lymphomas, ADC measurements typically yield very low values (see below).

As a general rule, PET/CT is uncommonly used for the work-up of mandibular lesions. Nevertheless, it may be employed as a complementary examination for the staging of malignant tumours invading the mandible, such as SCC of the oral cavity with secondary mandibular invasion, in primary intraosseous SCC or in mandibular lymphoma. In cases of metastases to the mandible, PET/CT may reveal the location of the primary tumour - if unknown - or may effectively show involvement of multiple organs. The most commonly used radiotracer is ${ }^{18} \mathrm{~F}$-fluorodeoxyglucose (FDG). FDG is a glucose analogue that is taken up by metabolically active tumour cells using facilitated glucose transport. In clinical routine, quantification of tracer uptake is performed using the standardised uptake value (SUV). High SUVs reflect high glucose metabolism mainly seen in aggressive tumours (typically SCC and lymphoma), while lower SUV values are rather seen in slowly growing, less aggressive tumours, in tumours with large areas of necrosis or in inflammatory conditions. The recent implementation of integrated hybrid PET/ MRI systems in clinical head and neck oncology [8] holds promise as it combines morphological, functional and molecular information at the same time, thereby providing additional diagnostic gain. However, research into the potential clinical role of PET/MRI in comparison with PET/CT, MRI with DWI or the combination thereof, is still ongoing.

This review article is based on the retrospective evaluation of 11,725 panoramic radiographs seen during a period of 6 years at our institution. From a practical point of view, radiolucent mandibular lesions can be divided into lesions with welldefined borders and lesions with poorly defined borders.

\section{Radiolucent lesions with well-defined borders}

Radicular cyst

Odontogenic cysts are true cysts arising from the epithelium left over from tooth development. Radicular cysts, also called periapical cysts or apical periodontal cysts, are the most common odontogenic cysts $[1,2]$. Tooth infection may lead to necrosis of the pulp cavity and may spread to the tooth apex with ulterior development of a periapical granuloma or periapical abscess. The latter may subsequently give rise to a radicular cyst. Radicular cysts arise from epithelial cell rests of the periodontal ligament, which are stimulated by the inflammatory products. Most often, radicular cysts are asymptomatic. They may be seen in all age groups, however, more often between 30 and 60 years of age and are typically associated with a non-vital tooth. Imaging features are straightforward and include a unilocular periapical lesion with well-defined, sclerotic borders in close vicinity of the apical portion of the root of a non-vital tooth. No contrast material enhancement is seen on CT or MRI. In atypical cases with latero-dental location, $\mathrm{CT}$ or $\mathrm{CBCT}$ is very helpful for the correct diagnosis (Fig. 1). Treatment options include apical surgery, tooth extraction and endodontic treatment.

\section{Residual cyst}

Residual cysts are periapical cysts retained in the jaw after surgical removal of a non-vital tooth $[1,3]$. Residual cysts are common and have similar clinical and radiological features as radicular cysts. However, there is always a missing tooth (Fig. 2). Most residual cysts are less than $1 \mathrm{~cm}$ in size. Occasionally, enlarging cysts may cause displacement of the adjacent teeth, as well as bone expansion.

\section{Dentigerous cyst}

Follicular cysts, also called dentigerous cysts or pericoronal cysts, are the second most common odontogenic mandibular cysts and the most common developmental cysts of odontogenic origin. They are typically seen in patients aged 20-40. Once fluid accumulates between the enamel organ

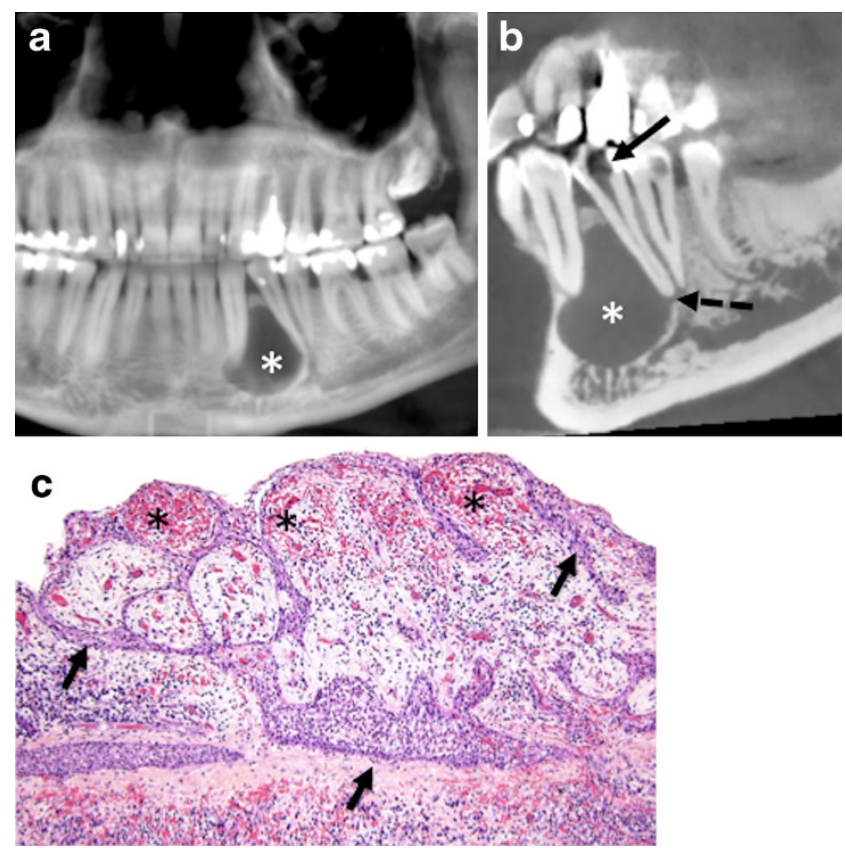

Fig. 1 Radicular cyst. a OPT. Unilocular radiolucent lesion with welldefined borders (asterisk) displacing teeth 33 and 34. b CT, sagittal oblique 2D MPR with bone windows. Cystic lesion surrounding the root (dashed arrow) of the non-vital tooth 34. Associated large caries with pulpal necrosis (arrow). c Histology (haematoxylin-eosin stain, original magnification $20 \times$ ): fibrous connective wall containing a dense chronic inflammatory infiltrate and congested vessels (asterisk) lined by irregular non-keratinised stratified squamous epithelium. Spongiotic epithelium (arrows) penetrated by neutrophils 
Fig. 2 Residual cyst. a OPT. Unilocular well-defined lesion with sclerotic borders (arrow) and missing tooth 36 above. $\mathbf{b}$ Histology (haematoxylin-eosin stain, original magnification $20 \times$ ): non-keratinised stratified squamous epithelium (arrow) with mild inflammation
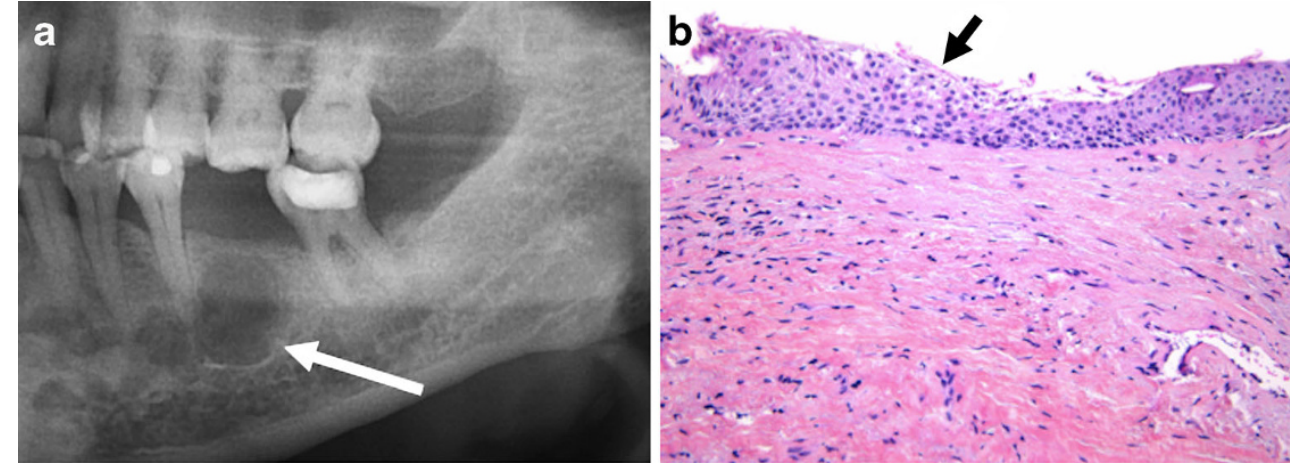

remnants and the tooth crown, a cyst forms around the crown of the unerupted tooth (typically the lower third molar). Imaging features of follicular cysts are characteristic and include a unilocular, well-defined radiolucent lesion with sclerotic borders around an unerupted tooth crown (Fig. 3). Small follicular cysts may be difficult to differentiate from a normal dental follicle. It has been suggested that when the distance between the crown and the dental sac is superior to $3 \mathrm{~mm}$, the diagnosis of a follicular cyst should be made. The cyst is most often attached to the neck of the unerupted and displaced tooth, and the crown generally protrudes into the cyst while the roots remain outside the lesion (Fig. 3). Follicular cysts may become very large [9] and may remodel the mandible, predisposing the patient to pathological fracture and infection. They may thin the cortical layer of the mandible and may insinuate around the mandibular canal, making surgical excision risky. $\mathrm{CT}$ and $\mathrm{CBCT}$ are used to determine the relationship of the cyst to the mandibular canal prior to surgery and to assess cyst contents, as well as integrity of the cortical rim (Fig. 3). MRI is indicated only in atypical cases to differentiate follicular cysts from other cystic mandibular lesions [5]. Follicular cysts will often be found incidentally in patients undergoing head and neck MRI for other indications. MRI findings include variable signal intensity on $\mathrm{T} 1$ due to variable protein content within the cyst, high signal on $\mathrm{T} 2$ and occasionally slight enhancement of the thin cyst wall. Although most cysts show no significant FDG uptake on PET/CT, mild tracer uptake may be seen in the presence of infection or inflammation [10]. Treatment options include enucleation in smaller lesions and marsupialisation in larger cysts. Rarely, SCC (see "Pitfalls") or ameloblastoma may arise from the epithelium of the cyst wall [11-13]. Multiple follicular cysts are very rare and may be seen in cleidocranial dysplasia, muccopolysaccharidosis type 4 [9] and in the Gorlin-Goltz syndrome.

\section{Keratocyst}

Keratocysts, also called primordial cysts, keratinising cysts or keratocystic odontogenic tumours (KCOTs), are benign intraosseous tumours arising from the dental lamina [11].
They are lined by stratified keratinising epithelium. Due to their potentially aggressive, infiltrative behaviour, they have a high recurrence rate after surgery (up to $60 \%$ ) $[1,14,15]$. KCOTs are often asymptomatic. They are often misdiagnosed as periapical cysts, follicular cysts, lateral periodontal cysts or ameloblastoma [14]. According to the literature, KCOT have a periapical position in $33 \%$ of cases, a pericoronal position in $21 \%$ of cases, a lateral root position in $19 \%$ of cases and are not related to any dental structures in $27 \%$ of cases [14]. KCOTs essentially occur in the 2 nd and 3 rd decade in the posterior body or in the ascending ramus of the mandible. Lesions in the molar and premolar area or in the anterior mandible are less common. Men are affected more commonly than women, most KCOTs occurring in Caucasian populations of Northern European decent. As KCOTs may become very large, they tend to remodel, hollow and expand the mandible (Fig. 4). Smaller lesions tend to be unilocular, while larger lesions tend to be multilocular. KCOTs show welldelineated, sclerotic or scalloped borders, soft-tissue extension and daughter cysts. The adjacent teeth are only rarely resorbed; however, when this is the case, differentiation from ameloblastoma may be very difficult on OPT, CT or CBCT. As KCOTs contain a cheese-like material, they typically show soft tissue density (up to $50 \mathrm{HU}$ ) on CT (Fig. 4), while on MRI, due to the variable protein content, a low to high signal intensity may be seen on $\mathrm{T} 1$ and a heterogeneous signal on $\mathrm{T} 2$. $\mathrm{T} 2$ relaxation times in KCOT have been reported to be shorter than in ameloblastoma [5]. Weak enhancement of the uniformly thin and regular cyst walls is noted after injection of gadolinium chelates, thereby facilitating differentiation from ameloblastoma [5].

Multiple KCOTs are seen in the nevoid basal cell carcinoma syndrome (Gorlin-Goltz syndrome), the oral-facial-digital syndrome, the Ehlers Danlos and in the Noonan syndrome $[2,16]$. The Gorlin-Golz syndrome is an autosomal dominant disorder manifesting with mental retardation, multiple KCOTs (Fig. 5), basocellular carcinomas, bone defects, subcutaneous and falx cerebri calcifications. Patients typically have large calvaria, midface hypoplasia, high-arched eyebrows and hypertelorism. The treatment of choice in KCOTs is complete surgical removal of the lesion itself, including the commonly associated 

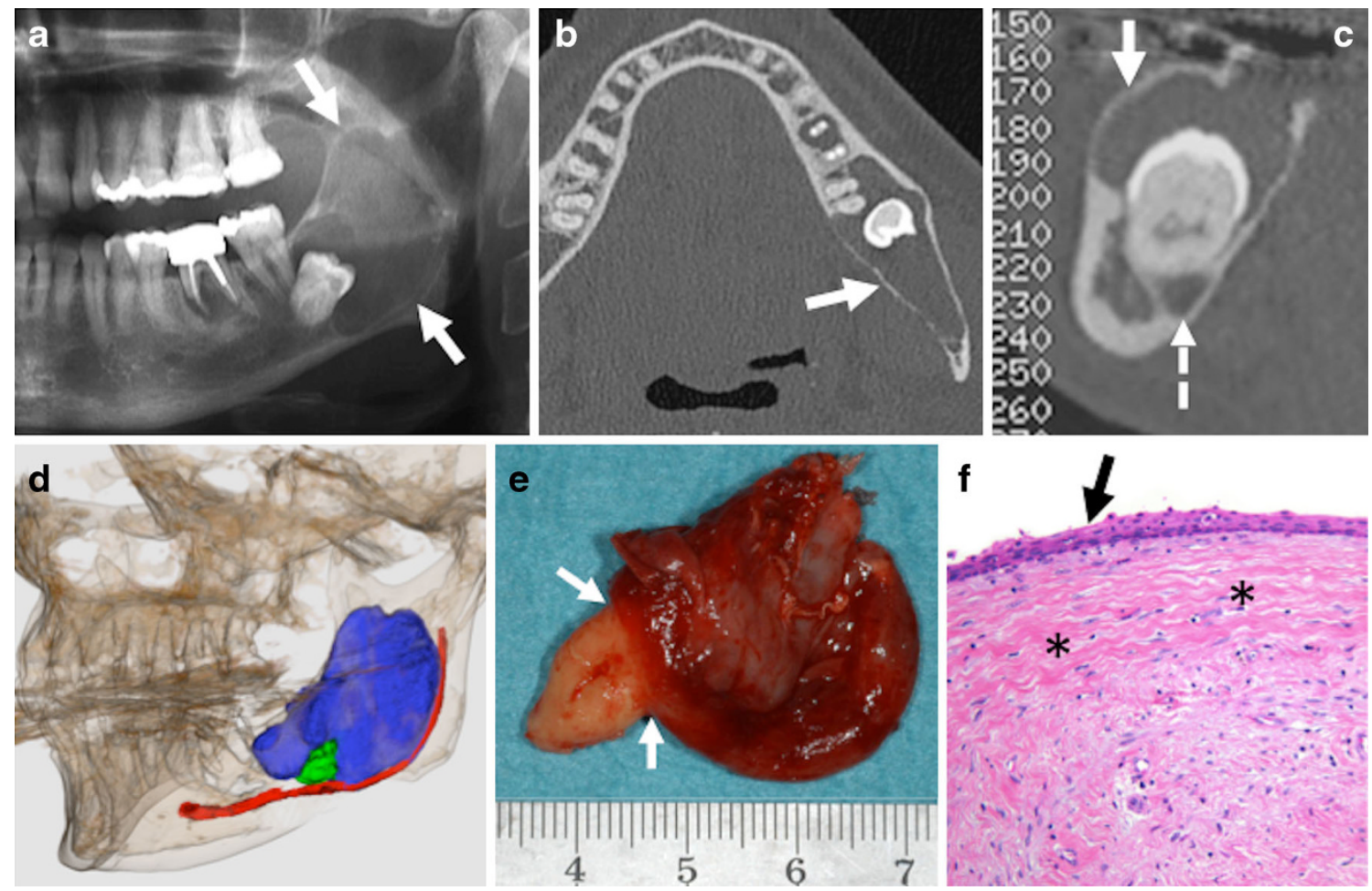

Fig. 3 Dentigerous cyst. a OPT, b axial CT with bone windows and c dentascan reconstruction. Unilocular well-defined radiolucent lesion (thick arrows) surrounding an unerupted tooth. Immediate vicinity of the mandibular canal (dashed arrow) to the impacted tooth. d Threedimensional reconstruction, lateral view showing the relationship of the cyst (blue), the unerupted tooth (green) and the mandibular canal (red).
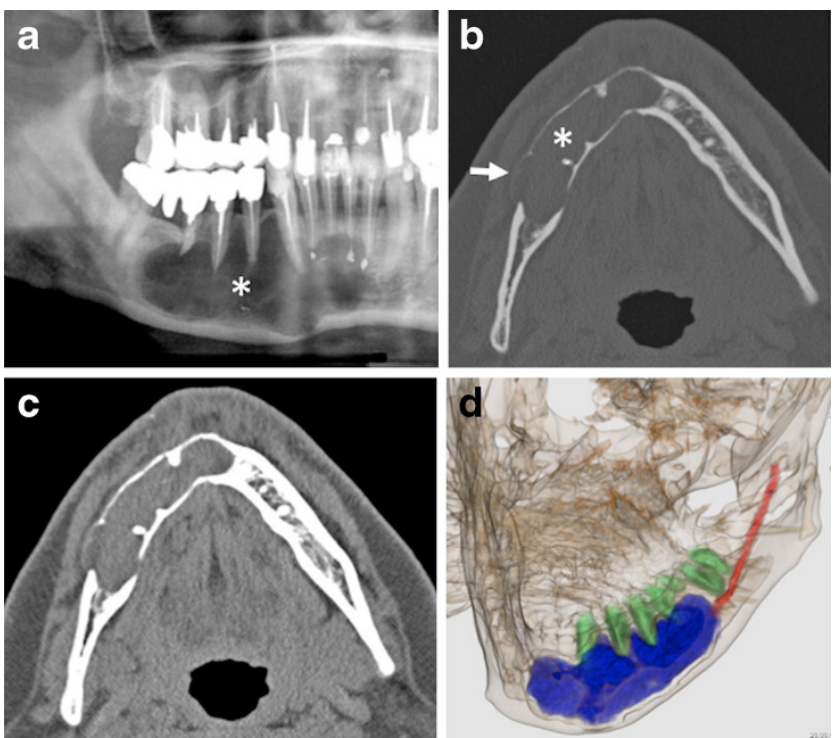

Fig. 4 Histologically proven KCOT. a OPT. Axial CT image with bone window (b) and soft tissue window (c). Multilocular well-defined radiolucent lesion (asterisk) with thin, sclerotic borders, cortical scalloping (arrow) and resorption of teeth roots. Cyst contents with attenuation values of $40-50 \mathrm{HU}$ mimicking solid tissue. d Posterior view of a 3D reconstruction showing the relationship between the keratocyst (blue), the partially resorbed teeth (green) and the mandibular canal (red). The mandibular canal can be seen only in the posterior mandible, as it is encased by the keratocyst
The cyst is attached at the level of the tooth neck and surrounds the crown. e Surgical specimen. Characteristic attachment of the cyst at the tooth neck (arrows). f Histology (haematoxylin-eosin stain, original magnification 20×): non keratinising thin epithelial lining (arrow) without rete pegs. Fibrous wall (asterisks) almost completely devoid of inflammatory cells

tooth. Careful clinical and radiological post-operative followup is essential due to the high recurrence rate.

\section{Ameloblastoma}

Ameloblastomas are benign but locally invasive, slowly growing odontogenic tumours arising from remnants of the dental lamina and dental organ or, less frequently, from the epithelial lining of an odontogenic cyst [12]. They represent $10 \%$ of all odontogenic tumours, most lesions being located in the mandible (posterior body and ramus region). Most tumours tend to occur during the 4th-6th decade and there is no sex predilection. Clinical features are non-specific and patients may complain of unilateral painless swelling. Very often, ameloblastomas are detected incidentally. Although classic amelobastomas do not have distant metastases, variants with metastatic behaviour despite histologically benign features (so-called "malignant ameloblastoma"), as well as tumours with histologically malignant features but without metastatic potential (so-called "ameloblastic carcinomas") have been described in the literature [3]. Ameloblastomas may be subdivided into four histological types: unicystic, multicystic, extraosseous, and desmoplastic [12]. The radiological appearance depends on the histological type and 
Fig. 5 Basal cell nevus syndrome. a OPG. Multiple mandibular and maxillary KCOTs (asterisks) associated with impacted teeth. b Intraoperative view showing cheese-like material within the angulomandibular lesion (arrow). c Surgical specimen showing the KCOT (asterisk) and the associated tooth (thin long arrow). d Histology (haematoxylin-eosin stain, original magnification $40 \times$ ): corrugated (dashed arrows) parakeratinised epithelium with distinct basal columnar cells with inverted polarity (arrows) and flat connective tissue interface
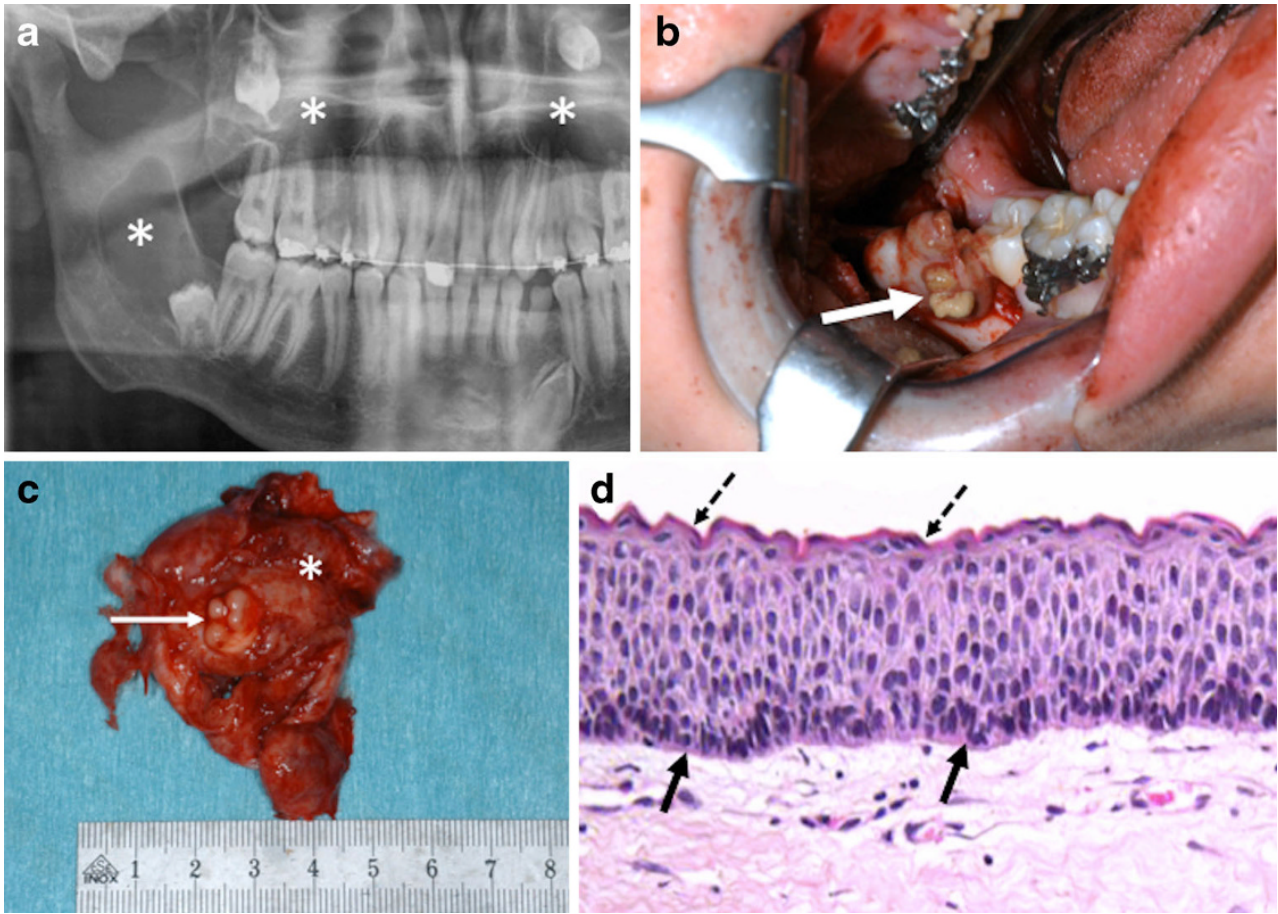

includes unilocular or multilocular radiolucent lesions ("soap bubble" or "honeycomb" appearance) with sclerotic borders, displaced adjacent teeth with root resorption and/or extensive bone expansion (Figs. 6 and 7). CT and MRI are used to assess tumour contents, integrity of the mandibular cortical rim, relationship to the mandibular canal and precise intraosseous and soft tissue extension (Figs. 6 and 7). In multicystic ameloblastoma, MRI reveals cysts with variable protein content. Typically, after injection of gadolinium chelates, MRI shows enhancement of solid nodular components, of irregularly thick septations and of papillary projections (Fig. 6). Although differentiation between benign and malignant ameloblastoma may be impossible with CT and MRI, a very high FDG uptake on PET/CT strongly suggests malignant ameloblastoma or ameloblastic carcinoma [17]. Treatment consists in complete surgical excision and careful postoperative follow-up with imaging is mandatory.

\section{Simple bone cyst}

The simple bone cyst (SBC), also known as solitary bone cyst, traumatic bone cyst, haemorrhagic cyst or idiopathic bone cavity is filled with serous or haemorrhagic fluid and is characterised by the absence of an epithelial lining. Therefore, SBC is not a true cyst but rather a pseudocyst. SBC of the mandible usually occurs secondary to trauma, typically after tooth extraction with subsequent intramedullary haemorrhage. Most SBCs occur before the age of 20 with female predominance [18]. In up to $75 \%$ of all cases, SBCs are seen in the marrow of the posterior mandible. Most lesions are asymptomatic and discovered incidentally on dental radiographs. SBC occurs in close proximity to a vital tooth and is not associated with bone swelling unless there is
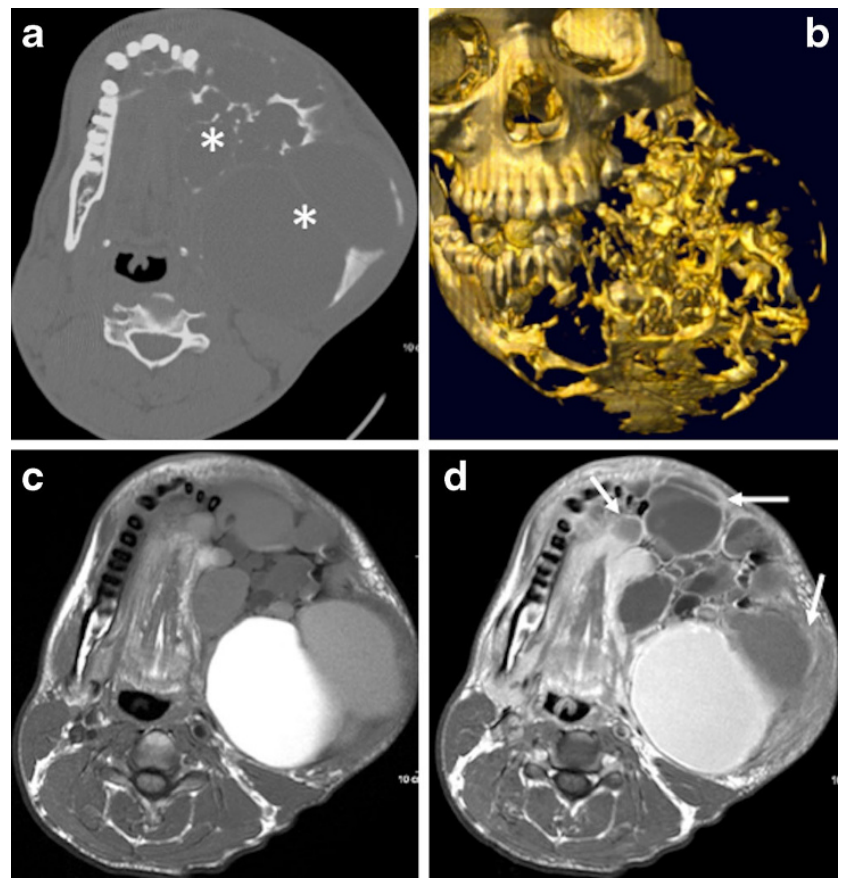

Fig. 6 Histologically proven multilocular ameloblastoma. a Axial bone window CT image. b Three-dimensional reconstruction, frontal view. c T1-weighted axial image. d T1-weighted axial image after injection of gadolinium chelates. Multilocular expansile radiolucency with characteristic "soap bubble" appearance (asterisks) and major facial deformation. Cystic components with variable signal intensity on the unenhnanced T1weighted image suggesting variable protein content. Note variable enhancement of solid components ranging from thin enhancing walls to thick enhancing solid portions (arrows) 
Fig. 7 Recurrent multilocular ameloblastoma. a OPT showing a well-defined, multilocular radiolucency (arrows) with sclerotic borders. b Resected specimen and $\mathbf{c}$ specimen radiograph clearly show bony expansion, destruction of the alveolar ridge and characteristic multilocular appearance. d Histology (haematoxylin-eosin stain, original magnification $20 \times$ ): follicular ameloblastoma with squamous metaplasia (red asterisks) within follicles (arrows). Largely fibrous stroma (black asterisks) containing scattered lymphocytes
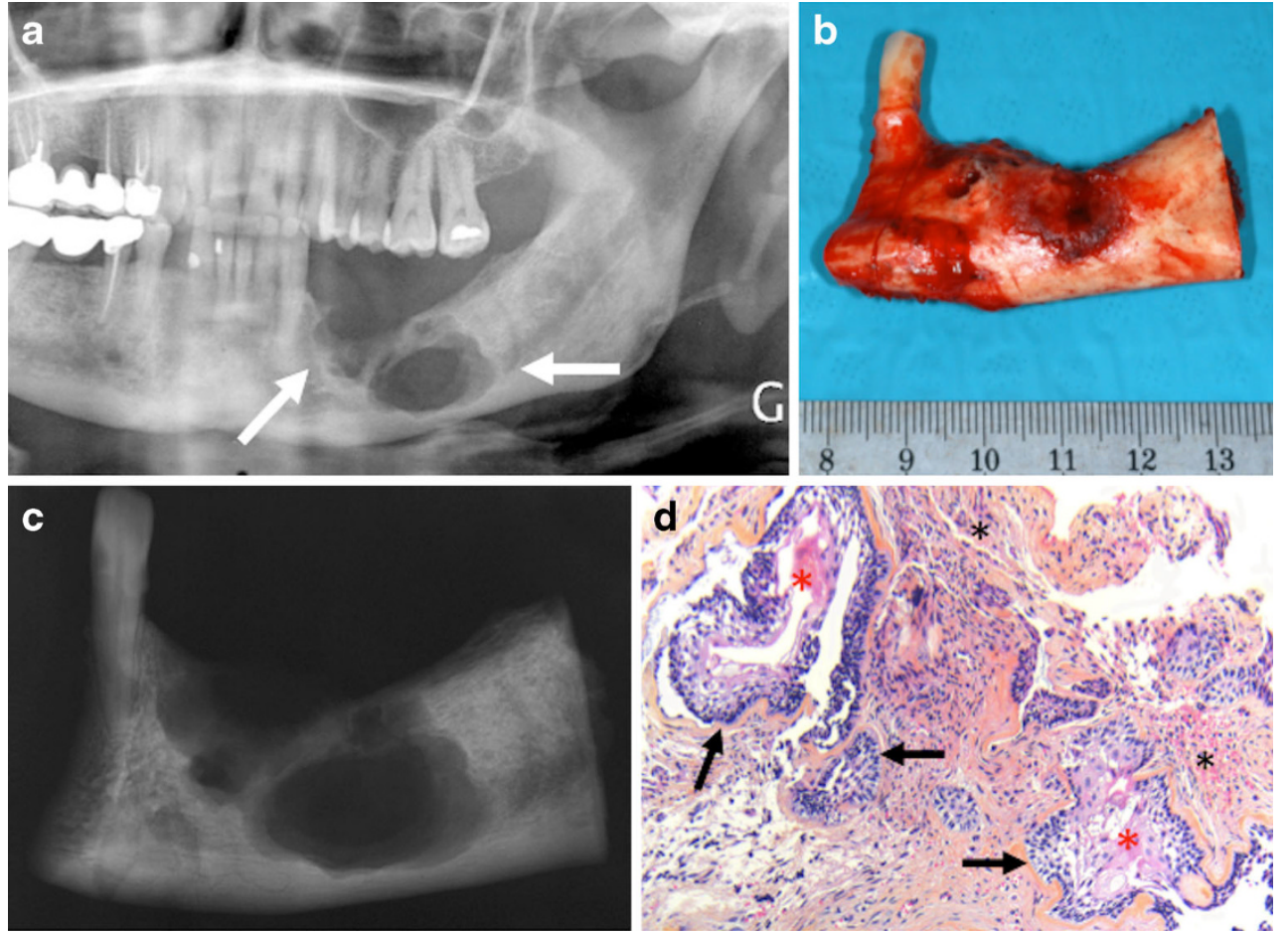

associated infection. Surgical exploration may be necessary in selected cases to exclude other unilocular radiolucent lesions, such as keratocysts. On imaging, most SBCs appear as unilocular, well-defined radiolucent lesions that vary in size. Rarely, poorly defined borders are observed. Extension to the cortical bone is rare and usually there is no tooth displacement [18]. The superior margins are irregular and scallop around teeth roots. CT and MRI can provide information on the haemorrhagic content but the density or signal intensity, respectively, may vary depending on the age of the haemorrhage. On MRI, the cysts display a homogeneous intermediate signal on $\mathrm{T} 1$, homogeneous high signal on $\mathrm{T} 2$, and no enhancement after injection of intravenous contrast media. Treatment consists of bone curettage, leading to bleeding with subsequent scar formation.

\section{Eosinophilic granuloma of the mandible}

Eosinophilic granuloma (EG) is a benign disease related to any of the three forms of Langerhans cell histiocytosis (LCH). Depending upon the number of lesions and lesion distribution, LCH comprises the following groups: unifocal LCH (also called EG), multifocal unisystem and multifocal multisystem. The combination of diabetes insipidus, lytic bone lesions and exophtalmus is known as Hand-Schüller-Christian triad, whereas the multifocal multisystem LCH is also called AbtLetterer-Siwe disease (with typical abdominal involvement and poor prognosis) [19]. LCH is caused by clonal proliferation of activated dendritic cells and macrophages [20]. Any bone can be affected, such as the skull, mandible, ribs and long bones, as well as any organ system (lung, skin, spleen, lymph nodes, central nervous system). Therefore, crosssectional whole-body imaging is essential for the initial work-up of patients with active disease and for posttherapeutic follow-up purposes.

The incidence of mandibular EG constitutes less than $10 \%$ of all LCH cases. LCH often affects males in the 1 st-3rd decade, although most cases are seen in children younger than 15 years of age. Mandibular involvement is seen preferentially in the body or angle (Fig. 8). Clinical presentation can be silent or non-specific including pain, swelling, fever, general malaise, gingival hypertrophy, ulcers of the buccal mucosa, limitation of mouth opening or tooth hypermobility and loosening. Pathological fracture may ensue. Radiologically, EG presents as a well-defined radiolucent lesion, with or without reactive sclerosis on OPT; however, an accompanying periosteal reaction is typically seen on CT or CBCT. Occasionally, this periosteal reaction may show a sunburst appearance, suggesting a more aggressive biological behaviour. If the alveolar crest is invaded, a "scooped out" appearance is seen; when the alveolar bone is destroyed, a "floating tooth" appearance is observed. EG is often associated with a soft tissue mass surrounding the mandible and invading the muscles of mastication. Morphological MRI findings include hypointense signal on $\mathrm{T} 1$, hyperintense signal on $\mathrm{T} 2$ and marked enhancement after intravenous gadolinium (Fig. 8). In our experience, $A D C$ values in EG are usually slightly higher than in malignant lesions $\left(\geq 1.2 \times 10^{-3} \mathrm{~mm}^{2} / \mathrm{s}\right.$ versus $\left.\pm 1 \times 10^{-3} \mathrm{~mm}^{2} / \mathrm{s}\right)$. In cases 

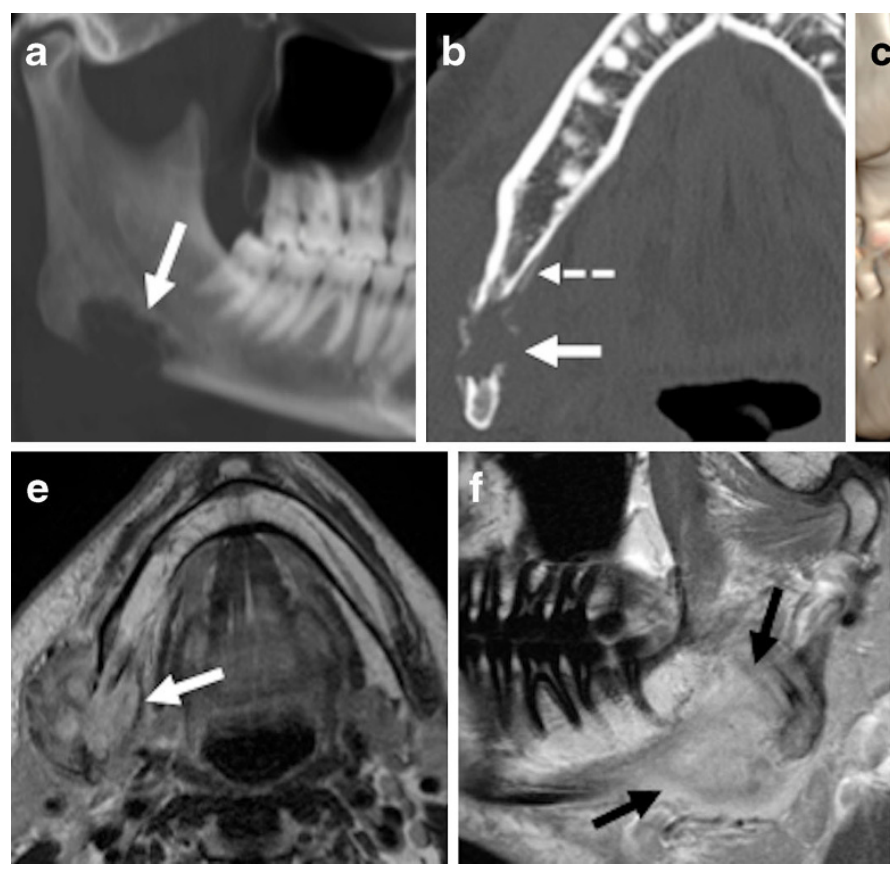

Fig. 8 Histologically proven eosinophilic granuloma. a OPT-like curved thick slab reconstruction, $\mathbf{b}$ axial CT bone window and (c) 3D reconstruction from CT data set show a well-defined radiolucent lesion (arrows) located in the angle of the mandible. Sharply delineated slightly sclerotic borders. Periosteal reaction (dashed arrow). d PET/CT showing that high FDG uptake within the posterior mandible and perimandibular soft tissues (arrow). SUVmean $=6, \mathrm{SUVmax}=9$. e Axial T2 and $\mathbf{f}$
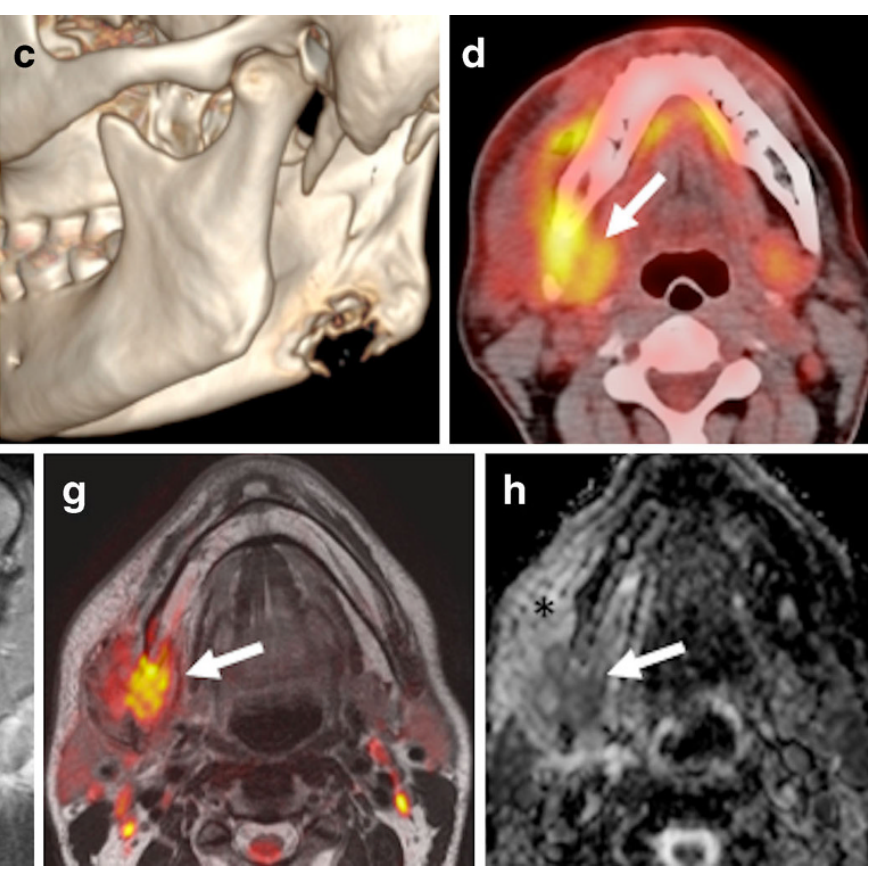

sagittal T1 after intravenous gadolinium show a large extraosseous component (arrows) with invasion of the masseter muscle. Close vicinity to the submandibulat gland. Note high signal intensity on T2 and strong enhancement after gadolinium. g Fused T2 and $b 1,000$ showing restricted diffusion. $\mathbf{h} \mathrm{ADC}$ map showing a moderately low $\mathrm{ADC}$ value $(\mathrm{ADC}=$ $1.21 \times 10^{-3} \mathrm{~mm}^{2} / \mathrm{s}$, arrow) within the lesion. Note inflammatory oedema around the lesion with high $\mathrm{ADC}$ values (asterisk)

with atypical periosteal reaction and poorly defined margins, the differential diagnosis includes malignant tumours, such as osteosarcoma, Ewing sarcoma or metastases. Nevertheless, well-defined margins on CT, the high signal intensity on T2, the strong enhancement, the large area of inflammatory oedema and the moderately high $\mathrm{ADC}$ values rather suggest a lesion with benign histology (Fig. 8). The value of MRI in patients with EG lies in its high sensitivity especially when used in conjunction with DWI [20]. PET/CT has been shown to provide valuable information for the detection of EG lesions due to their high FDG uptake (Fig. 8) and it has been suggested that hybrid PET/MRI may play a pivotal role for the primary investigation of paediatric histiocytosis allowing accurate detection of multifocal multiorgan involvement and for monitoring treatment response [20]. Despite the relatively characteristic imaging findings, definitive diagnosis of EG is made on samples obtained by biopsy. Treatment modalities include surgery, chemotherapy and intralesional injection of corticosteroids.

\section{Giant cell granuloma}

Giant cell granuloma (GCG) is a benign but occasionally aggressive proliferative intraosseous lesion with fibrous tissue, haemorrhage and haemosiderin deposits, as well as characteristic osteoclast-like giant cells. GCG is a rare lesion occurring preferentially in young girls or women $[1,21]$. The posterior mandible is affected more often than the anterior mandible. Multifocal involvement is seen in hyperparathyroidism, cherubism or Noonan syndrome [21, 22]. The most common clinical features are pain, swelling, facial asymmetry and paresthesia. Differential diagnosis includes giant cell tumour, radicular cyst, ameloblastoma, odontogenic tumour and fibrous dysplasia. The typical radiological appearance is that of a multilocular (less often unilocular) well-defined radiolucent lesion. However, ill-defined lesions have also been reported. In some cases, bone may be expanded or teeth can be displaced or resorbed. CT, CBCT and MRI are useful for describing bony involvement and for evaluating extension into the adjacent soft tissues. On MRI, GCG has a homogeneous or slightly heterogeneous intermediate signal on T1, T2 and STIR (short TI inversion recovery) and shows moderate to strong contrast enhancement after administration of gadolinium. However, it is important to note that only very few data regarding the imaging characteristics of GCG are currently available in the literature. The treatment of choice is surgery (enucleation and curettage in well-defined GCG or en bloc resection in aggressive GCG) and medical treatment (intralesional corticosteroid or calcitonin injections) [21]. Recurrence may occur in up to $15 \%$ of cases. 


\section{Radiolucent lesions with ill-defined borders}

\section{Osteomyelitis}

Despite the introduction of antibiotics and improved medical care, osteomyelitis of the jaws is still relatively common. The mandible is affected more often than the maxilla. Depending on the clinical course, osteomyelitis of the mandible can be classified into three forms: acute, secondary chronic and primary chronic $[23,24]$. The acute and secondary chronic forms are manifestations of the same disease entity separated by the arbitrary time limit of 1 month. These two forms are typically caused by bacterial infection in the setting of pulpal or periodontal infection, mandibular foreign bodies, sepsis or trauma. In dental infection, pulpal infection extends into the bone marrow and there is compression of blood vessels from periapical lesions. Acute forms present with severe symptoms such as pain, swelling, fever, lymphadenopathy or a mobile tooth sensitive to percussion. They may also present with paresthesia in the lower lip (V3 nerve), trismus or fistula with pus and - in advanced stages - osteomyelitis may also be revealed by pathological fractures. The chronic forms are clinically silent but often include painful periods. Predisposing factors are diabetes, immunosuppression, radiotherapy and bisphosphonates.
While the diagnosis of osteomyelitis can be made clinically in many cases, imaging is especially helpful to define the extent and assess the severity of the lesion. The radiological appearance of osteomyelitis depends on the stage of disease. It consists of an ill-defined osteolytic lesion (Fig. 9), with bone sequestra, and - in subacute cases - is often associated sclerosis and periosteal new bone formation. CT is helpful to analyse the bone structure, to search for associated abscesses and to identify soft tissue extension (myositis, fasciitis, cellulitis). MRI findings in osteomyelitis are similar to those in osteoradionecrosis (Fig. 9) and the differentiation between the two entities is based on the clinical context. MRI is more sensitive than CT in detecting marrow and soft tissue involvement [25]. In addition, MRI allows earlier diagnosis of osteomyelitis than $\mathrm{CT}, \mathrm{CBCT}$ or conventional radiographs. In general, the disease extent appears larger on MRI than on CT; therefore, a combined MRI and CT assessment is most often required pre-operatively. Osteomyelitis lesions show low signal on T1, and high signal on T2 and STIR images due to oedema of the bony marrow; variable degrees of contrast enhancement of the marrow itself and of adjacent soft tissues are equally observed (Fig. 9). The signal intensity of bone sequestrae, however, is very low on all sequences. Treatment consists of a combination of antibiotics and surgical debridement.
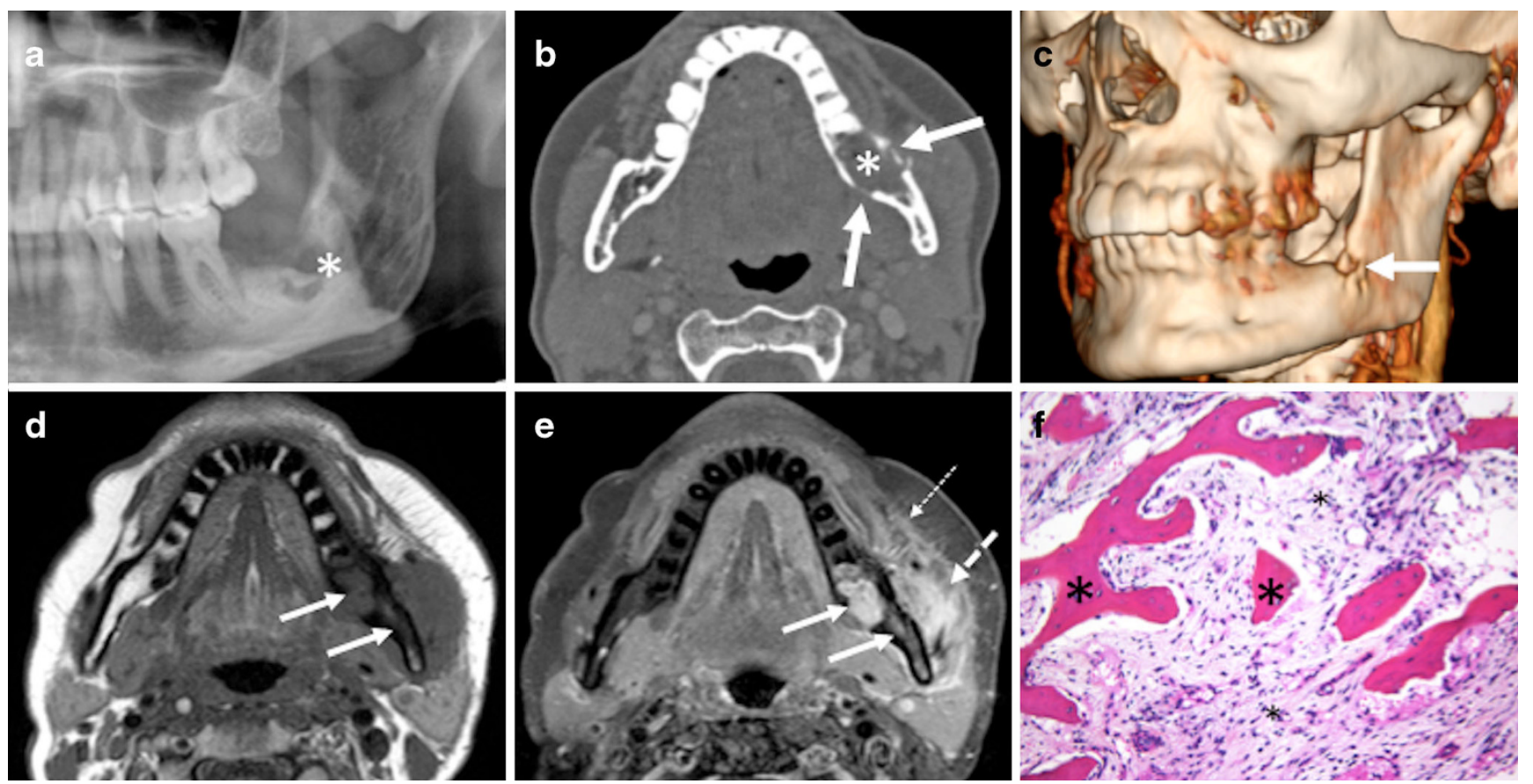

Fig. 9 Osteomyelitis after tooth extraction. a OPT. b Contrast-enhanced, axial CT image with bone window settings. c Three-dimensional reconstruction. Ill-defined osteolytic area (asterisks) extending into the ascending ramus of the mandible, with cortical destruction (arrows in $\mathbf{b}$ and $\mathbf{c}$ ). d Axial T1-weighted image before (d) and after (e) injection of gadolinium chelates. Hypointense signal of the mandible (arrows in d) due to marrow oedema and strong enhancement (arrows in e) due to hyperaemia. Myositis of the masseter muscle (thick dashed arrow) and streaky enhancement of the subcutaneous fat and platysma muscle (thin dashed arrow) suggesting a phlegmon. f Histology (haematoxylin-eosin stain, original magnification $20 \times$ ): non-viable trabeculae (large asterisks) with bone resorption and destroyed osteoblasts. Inflammatory infiltrate (small asterisks) with neutrophils and lymphocytes as well as increased vascularisation within the fatty marrow 


\section{Bisphosphonate osteonecrosis}

Bisphosphonate treatment, similar to radiotherapy, may cause bone necrosis. Bisphosphonates are widely used for the treatment of cancer-related symptoms. Their benefits consist in reducing hypercalcaemia, limiting progression of bone lesions and preventing pathological fractures in bone metastases from breast, prostate and lung cancer, as well as multiple myeloma [26]. In short, bisphosphonates are widely indicated to improve the quality of life in a large number of patients. Bisphosphonate-related osteonecrosis of the jaw (BRONJ) was defined by the American Association of Oral and Maxillofacial Surgeons (AAOMS) based on three criteria: past or current bisphosphonate treatment, osteonecrosis lasting for at least 8 weeks and no history of radiotherapy to the jaw. [26]. BRONJ most often occurs in the mandible rather than the maxilla, especially in the molar region, and is frequently triggered by a pre-existing focal lesion. Clinical features include painful swelling, signs of infection (fever, oozing pus, abscess and soft tissue inflammation) and a mobile tooth sensitive to percussion. Occasionally, there is paresthesia in the lower lip (whenever V3 is affected) or trismus. Imaging features of BRONJ are the same as for osteoradionecrosis (Fig. 10). MRI can show signal changes on T1 and T2 images according to the stage of the disease. Although radiological findings are easily identified, they are non-specific and the final diagnosis is made by combining imaging findings with the clinical context [27].

\section{Osteoradionecrosis}

Osteoradionecrosis (ORN) is a serious uncommon complication (2.6-15\%) of radiotherapy in head and neck cancer patients. It usually occurs 5-15 years after radiotherapy. The risk of developing ORN increases with poor oral hygiene, alcohol and tobacco use after radiotherapy, tooth extraction or periodontal disease prior to radiotherapy: ORN is also related to the nutritional status of the patient. ORN preferentially affects the molar, premolar and retromolar region. Depending on the radiation portal and the administered radiation dose, osteoradionecrosis may affect the mandible unilaterally or bilaterally. Combined treatment options (radiation therapy, surgery and chemotherapy) appear to predispose to ORN [28]. Clinical manifestations include pain, limitation of mouth opening and deep ulcerations with denuded bone. In advanced stages, trismus, fistulas and pathological fractures have also been reported. CT usually reveals a patchy radiopaque-radiolucent ill-defined lesion with cortical bone destruction, usually without periosteal reaction (Fig. 11). Typical MRI findings are a mixture of marrow oedema (increased signal on T2) and marrow sclerosis (reduced signal on T2) with fragmentation of bone and sequestration (very low signal on
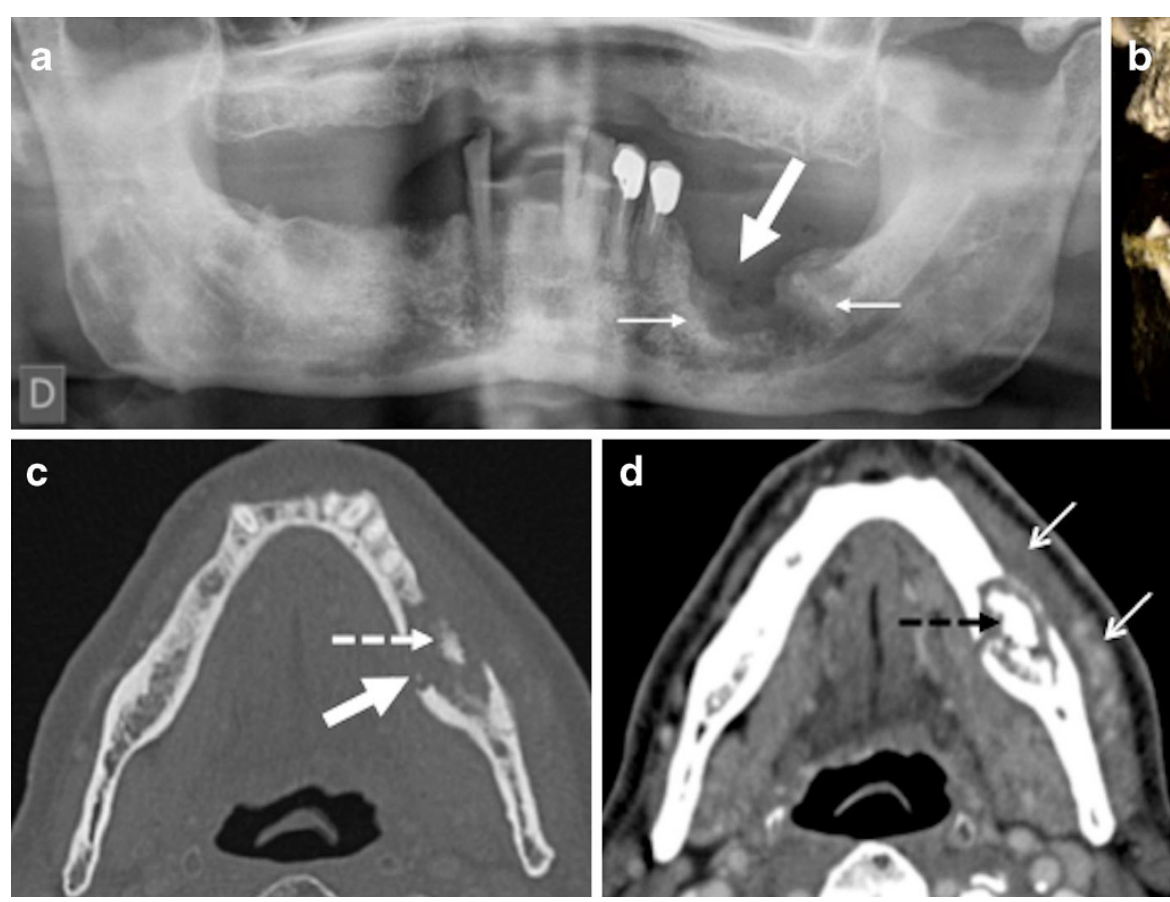

Fig. 10 Biphosphonate osteonecrosis. a OPT; b 3D reconstruction anteo-lateral view. Contrast-enhanced axial CT image with bone window settings (c) and soft tissue settings (d). Poorly defined osteolytic lesion of the left mandibular body (thick white arrows) with partly sclerotic margins (thin arrows in a) and large osseous defect (red arrows in b). Bone
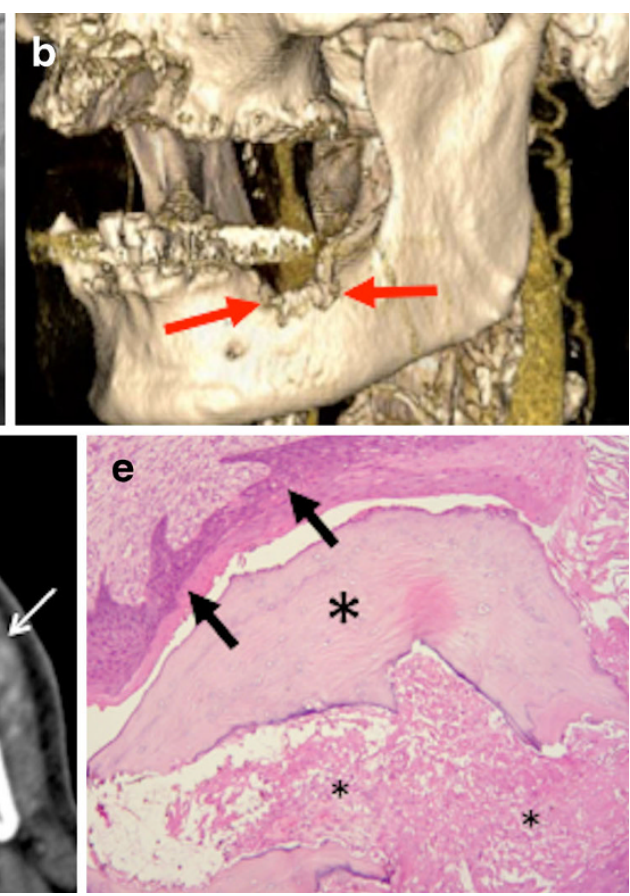

sequestra (dashed arrows). Associated subperiosteal phlegmon and myositis of the platysma (open arrows). e Histology (haematoxylin-eosin stain, original magnification 20×): necrotic bone (large asterisk) with inflammatory infiltration of the fatty marrow (small asterisks). Gingival mucosa (arrows) 

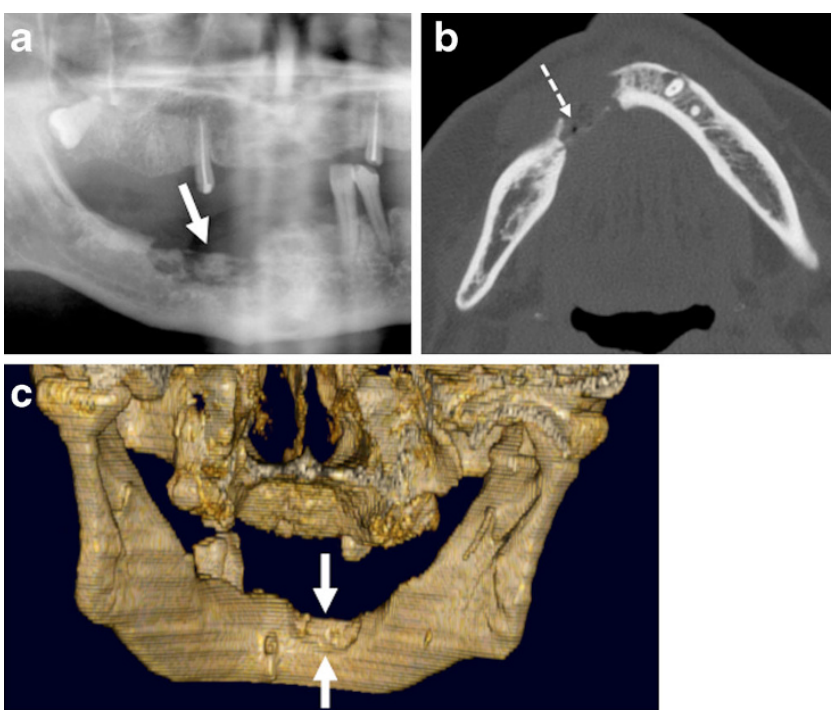

Fig. $11 \mathrm{CT}$ aspect of osteoradionecrosis 5 years after radiotherapy of a floor of the mouth SCC. a OPT. b Axial bone window CT image. c Three-dimensional reconstruction, posterior view obtained after removing the bony structures of the cervical spine. Characteristic osteolytic bone defect (arrows) with poorly defined partly sclerotic margins and soft tissue ulceration (dashed arrow). Follow-up confirmed absence of tumour

all sequences), as well as contrast enhancement after injection of gadolinium (Fig. 12). The differential diagnosis includes tumour recurrence, which can be differentiated from ORN with DWI (high ADC values in necrosis as opposed to low ADC values in tumours). The presence of a mass lesion within soft tissues does not necessarily suggest a tumour, as it may also be caused by a phlegmon, abscess or areas of fibrosis [29]. Treatment is both medical and surgical, but an important element in the management of ORN is prevention through avoidance of risk factors. The choice of treatment must take key factors, such as defect size, patient symptoms, and aesthetic and functional impairment into consideration. Medical therapy includes oral care, local debridement, nutritional follow-up, topical creams and hyperbaric oxygen therapy. If medical treatment fails, surgical management is indicated. It consists of resection of the necrotic bone and reconstruction with myocutaneous flaps [30].

\section{Osteoblastoma}

Osteoblastoma is a mesenchymal well-vascularised benign bone tumour with production of osteoid; it involves the skull in only $10 \%$ of cases [31]. It is uncommon and has a predilection for the posterior mandible, affecting mainly young people with a mean age below 20 years. The clinical symptoms include pain and swelling. The radiological presentation comprises an ill-defined (rarely well-defined) radiolucent or mixed radiolucent-osteosclerotic lesion. The tumour usually measures less than $4 \mathrm{~cm}$ and may be partially calcified. The borders are typically coarse and sclerotic (Fig. 13). Osteoblastoma has an expansile growth pattern and may cause teeth displacement but typically never leads to fusion with cementoma, a feature allowing differentiation from cementoblastoma [31]. On MRI, osteoblastoma displays major contrast enhancement after injection of gadolinium chelates (Fig. 13). The main differential
Fig. 12 MRI aspect of osteoradionecrosis 2 years after chemoradiotherapy of a tonsillar SCC. a Axial T1-weighted image. b Contrast-enhanced sagittal oblique T1-weighted image and ADC map (small image in $\mathbf{b}$ ). Bilateral hypointensity of the bony marrow. Large osseous defect with cortical destruction on the left (arrow in a). Marked, nonspecific contrast enhancement, bony destruction and necrotic hypointense sequestrae (arrow in b). Note high ADC value (red circle) measured at $1.53 \times$ $10^{-3} \mathrm{~mm}^{2} / \mathrm{s}$ suggesting inflammation and absent recurrence. c Macroscopic resection specimen. The necrotic area is indicated by arrows. d Histology (haematoxylin-eosin stain, original magnification $40 \times$ ): necrotic bone trabeculae with inflammatory infiltrative changes in the bony marrow
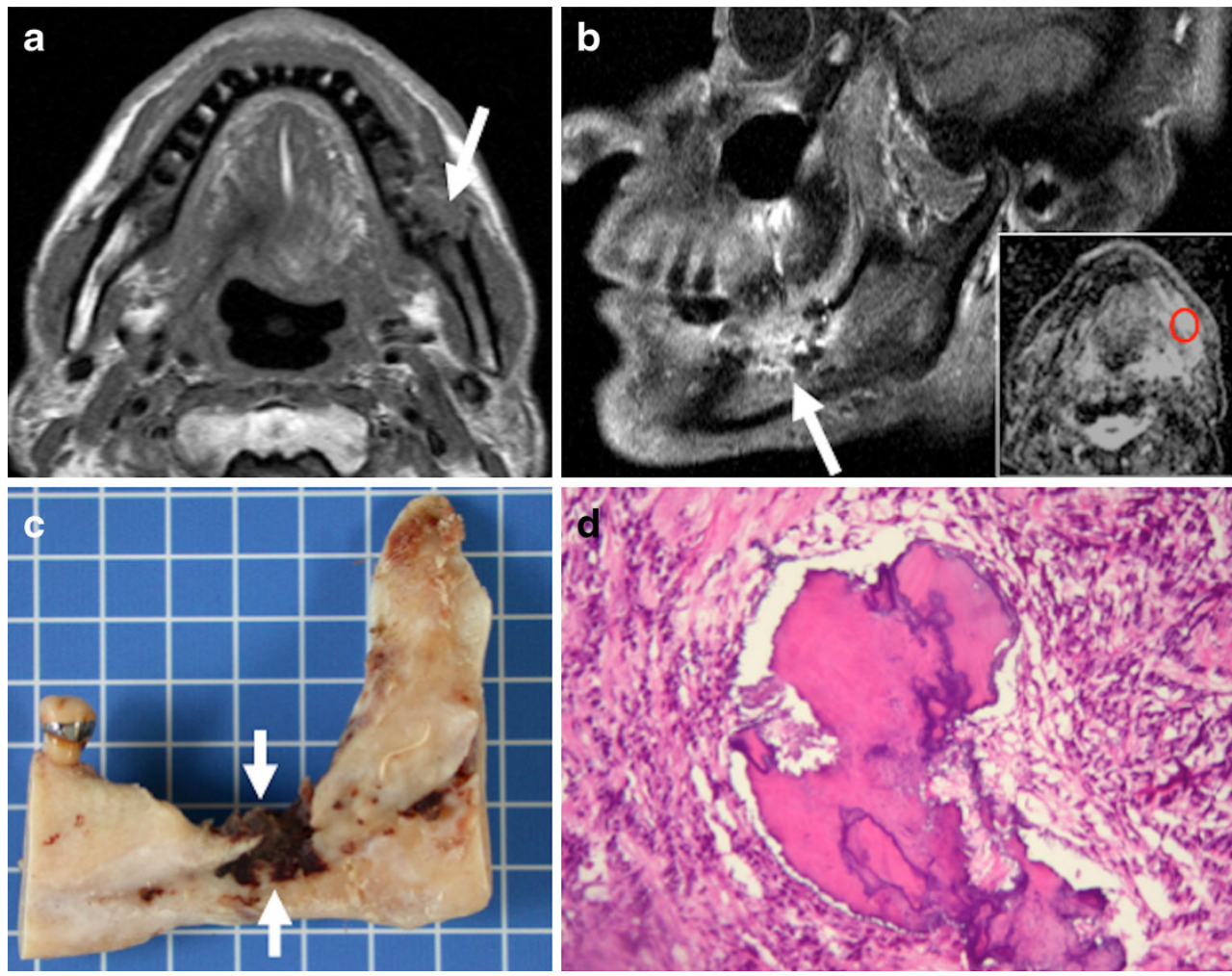
Fig. 13 Osteoblastoma. a OPT. b Axial CT with bone window. c Sagittal oblique contrastenhanced fat-saturated T1weighted image. Ill-defined radiolucent lesion (arrows in a and $\mathbf{b}$ ) with coarse sclerotic borders and calcifications (dashed arrow). Major contrast enhancement (arrow in c). d Histology (haematoxylin-eosin stain, original magnification $64 \times$ ). Osteoid and woven bone (asterisk) with plump osteoblastlike cells (arrows), interposed fibroblasts and some inflammatory cells
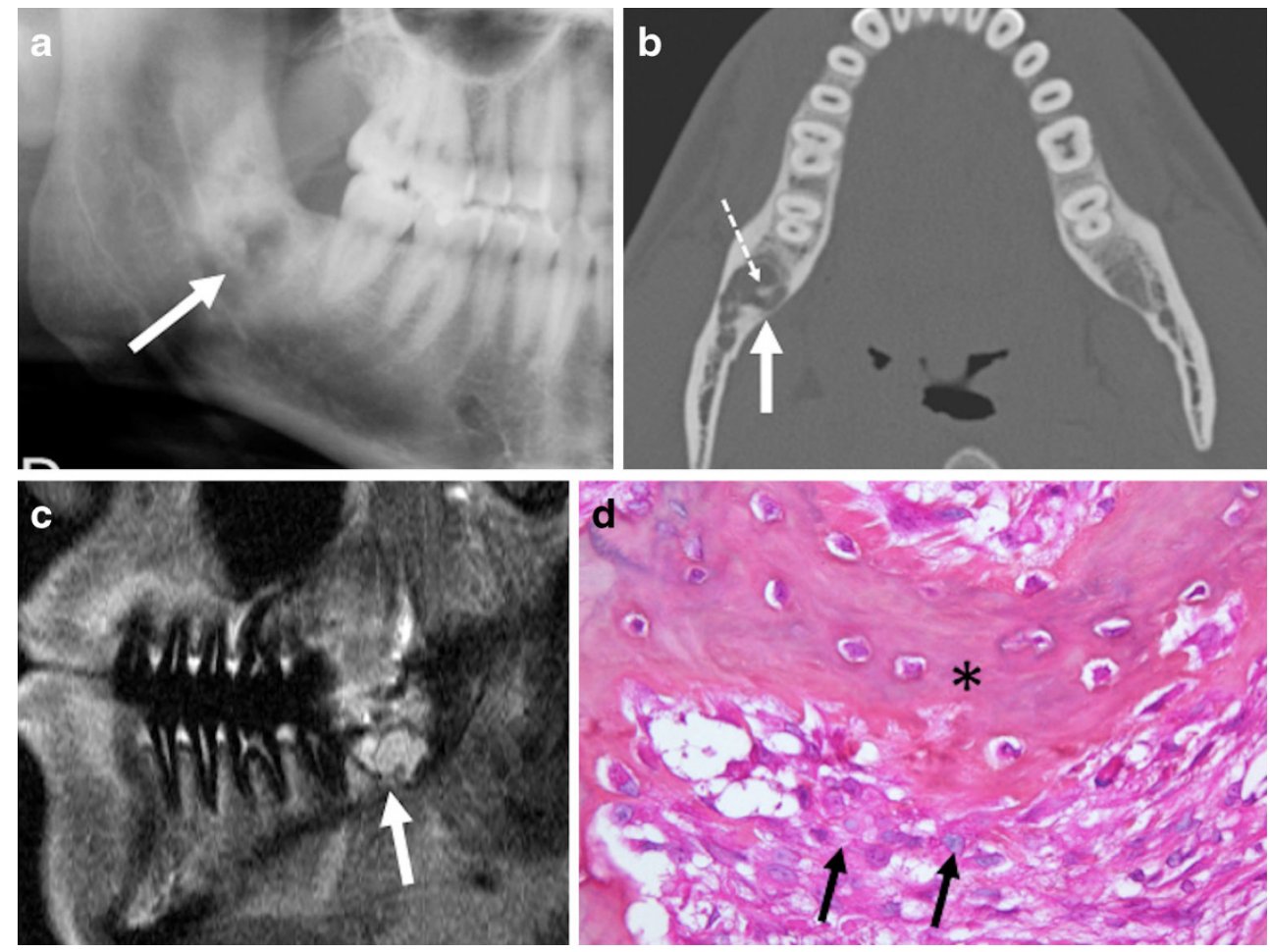

diagnosis includes osteoid osteoma, ossifying fibroma, periapical cemental dysplasia, fibrous dysplasia, as well as other fibro-osseous lesions. Osteoid osteoma typically presents with pain and a nidus can be identified at CT or CBCT. Ossifying fibroma most commonly occurs in the posterior mandible during the $3 \mathrm{rd}-4$ th decades [1]. Depending on the degree of calcification, the radiological appearance includes well-defined radiolucent, radiopaque or mixed opacity lesions [1]. Commonly, a radiolucent margin is seen on CT or CBCT, allowing differentiation from fibrous dysplasia. Periapical cemental dysplasia typically occurs in women during the 4th and 5th decades. It is the consequence of connective tissue proliferation within the periodontal membrane; therefore, the lesions are typically located in vicinity of tooth apices [1]. Initially, the lesions are radiolucent and with increasing maturity, there is advanced calcification. As opposed to mandibular osteoblastoma and osteoid osteoma, many fibro-osseous lesions do not cause pain despite their large size or associated teeth displacement. In addition, the age of presentation, the multiplicity of lesions and the relationship to tooth apices are further elements that help in the differential diagnosis. The treatment of choice in osteoblastoma is surgery and recurrence is rare [31]. Some cases of spontaneous regression have been reported, especially in young patients.

\section{SCC with mandibular invasion}

SCC, also known as epidermoid carcinoma, is a malignant neoplasm arising from the mucosa of the oral cavity [32, 33].
It is the most common tumour of the oral cavity and preferentially affects men over the age of 50. SCC most often occurs in the mucosa overlying the posterior mandible, and it is related to the consumption of alcohol and tobacco. It has high metastatic potential. Clinical symptoms include pain, swelling, paresthesia (due to V3 invasion) and dental disorders. In advanced stages, SCC may be discovered in the context of a pathological fracture. The radiological appearance is that of an aggressive soft-tissue lesion with invasion of the floor of the mouth, alveolar ridge or retromolar trigone [33]. In advanced stages, secondary bone invasion can occur leading to the appearance of an ill-defined radiolucent lesion of the mandible on conventional X-rays; in advanced lesions, the "floating teeth" sign may be equally observed due to extensive mandibular infiltration. On MRI, SCC displays a low to intermediate signal on T1, a moderately high signal on T2 and STIR sequences, and a moderate enhancement after injection of contrast media (Fig. 14). ADC values are typically low (usually around $1-1.1 \times 10^{-3} \mathrm{~mm}^{2} / \mathrm{s}$ ). Whenever large areas of necrosis are present within the tumour, ADC values may, however, be higher. This is particularly true in necrotic lymph node metastases (Fig. 14). Lymph node metastases in SCC of the oral cavity are common, especially in level I and II nodes. On FDG PET/CT or PET/MRI, SUV values are typically high due to the high glucose metabolism (Fig. 14). The final diagnosis and the pre-therapeutic work-up in SCC of the oral cavity are made by a combined clinical, imaging and histological work-up. The main role of imaging consists in precisely depicting deep tumour spread, as well as detecting lymph 


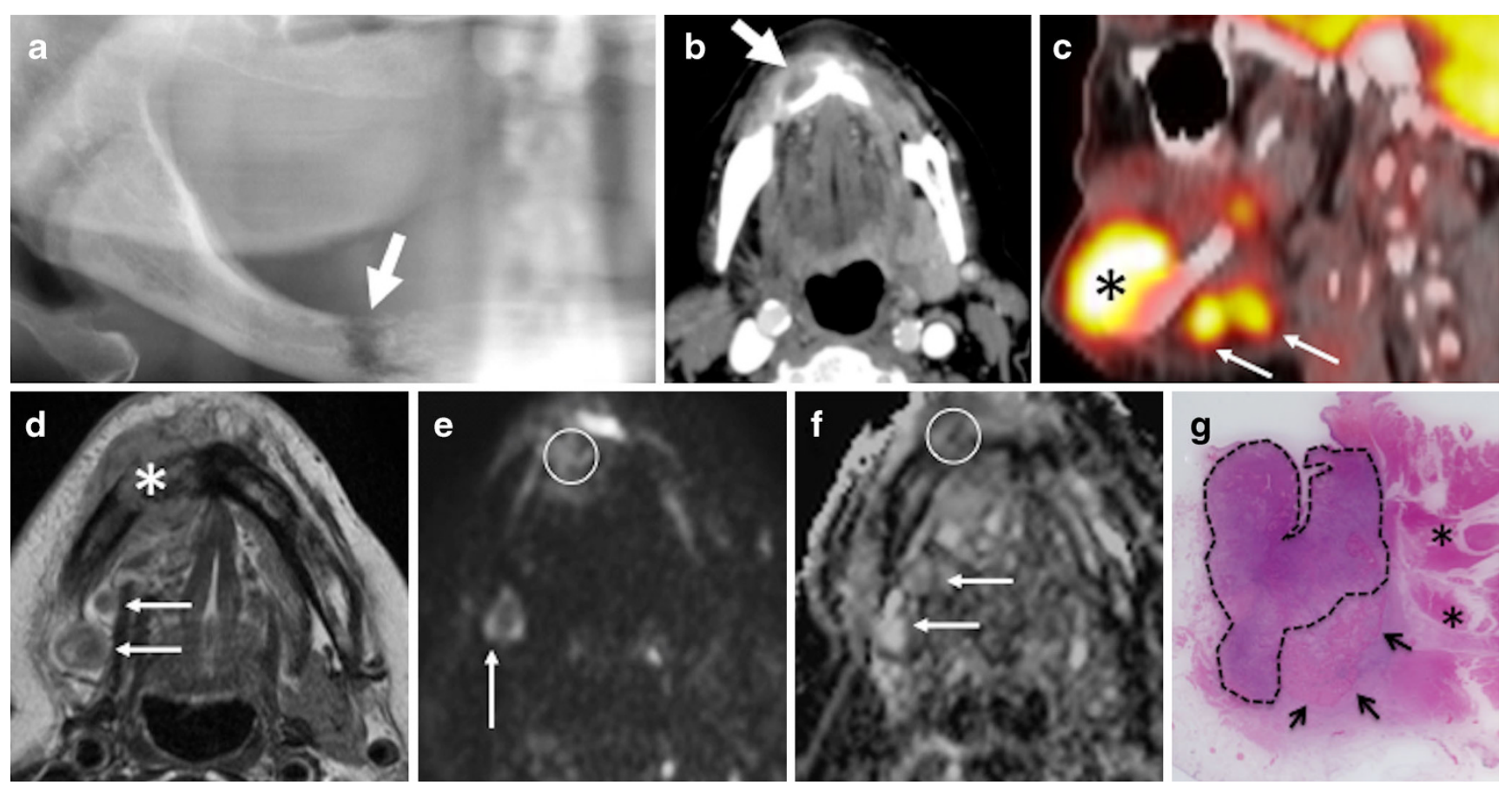

Fig. 14 SCC with mandibular invasion. Precise pre-operative assessment with MRI and PET/CT. a OPT. Poorly defined bony destruction (arrow) of the edentulous mandible. b Contrast-enhanced axial CT. Aggressive soft-tissue lesion (arrow) with secondary bone invasion. c Sagittal oblique PET/CT image. High metabolism of the tumour (black asterisk, SUVmean $=18, \mathrm{SUVmax}=22$ ) and of two metastatic level I lymph nodes $($ arrows, SUVmean $=10$, SUVmax $=15)$. d T2-weighted axial image. Tumour (asterisk) with marrow invasion and extensive infiltration of the floor of the mouth (dashed arrow). Note two metastatic level I lymph nodes (arrows). e The $b 1,000$ and $\mathbf{f}$ ADC map show restricted diffusion within the tumour (circle, $\mathrm{ADC}=0.98 \times 10^{-3} \mathrm{~mm}^{2} / \mathrm{s}$ ). Variable ADC values within the metastatic lymph nodes due to the presence of necrosis (arrows in $\mathbf{f}$ ). $\mathbf{g}$ Sagittal histological whole-organ slice of the resected specimen. Tumour (dashed black line) invading the mandible (black arrows) and the muscles (asterisk) of the floor of the mouth. The histological slice has the same orientation as (c) node and distant metastases. Surgery with or without radiotherapy is the treatment of choice. Prognosis depends on the histological type and on the presence or absence of lymph node metastases.

\section{Metastases}

Metastases to the jaw are an uncommon entity, affecting the mandible more often than the maxilla [34]. The most common primaries vary depending upon gender. Lung, prostate, kidney and liver tumours are the most common primaries in men, whereas breast, adrenal, gynaecological and colorectal tumours are the most common primaries in women [34]. Typical clinical symptoms include pain (Fig. 15), swelling, paresthesia, temporomandibular joint derangement, but in some cases metastases to the jaw are clinically silent and found incidentally. The radiological appearance includes illdefined radiolucent lesions with no periosteal reaction on conventional X-rays, CT and CBCT [35]. MRI reveals moderately hyperintense masses on T2-weighted and STIR images, hypointense signal on T1-weighted images and variable degrees of contrast enhancement. In general, the surrounding soft tissues lack relevant oedema and enhancement unless tumour extension beyond the mandible has occurred
(Fig. 15). On PET/CT, focal areas of increased FDG uptake are typically observed and measured SUVs are high (Fig. 15). Although the imaging aspect is not specific, in the presence of a patient with a history of cancer, the diagnosis of mandibular metastasis should be considered in the differential diagnosis first, particularly when the lesion shows no relationship to dental structures.

\section{Pitfalls}

Pseudolesions

\section{Stafne cyst}

Stafne cyst, also called static bone cavity or salivary gland inclusion defect, is a pseudocyst arising from bone remodelling caused by the adjacent submandibular gland. Therefore, it does not present any epithelial lining. Stafne cysts are often incidental findings, as patients are asymptomatic (Fig. 16). The lesions are more common in men than in women. The radiological aspect includes ovoid, well-defined radiolucent cortical defects on the lingual surface of the posterior mandible usually measuring less than $2 \mathrm{~cm}$. This location is typical 
Fig. 15 Condylar metastasis from adenocarcinoma. OPT (a) with osteolytic ill-defined lesion of the mandibular condyle (arrow). b Sagittal PET/CT image shows high metabolism with SUV $=12$ (arrow). c Axial, contrast-enhanced, fat-saturated T1-weighted image. The infiltrative, bulky lesion invades the condyle (arrow), the internal pterygoid muscle (dashed arrow) and part of the parotid gland (thin arrow). d Intraoperative view.

Extensive condylar involvement. e Histology (haematoxylin-eosin stain, original magnification $80 \times$ ): large atypical polygonal cells, some with several nuclei (arrow)
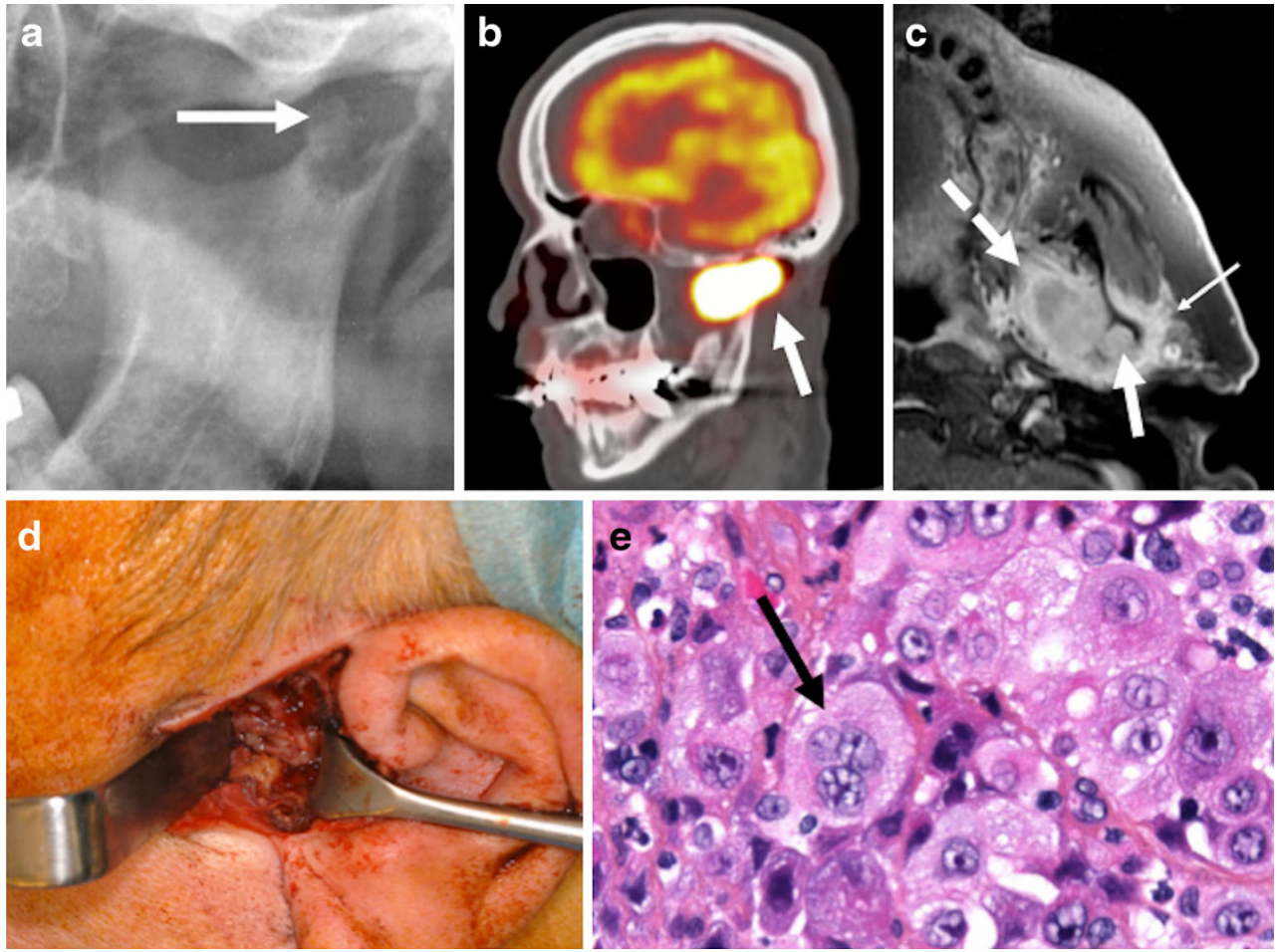

and the lesion contains fat or salivary gland tissue. An OPT is sufficient for diagnosis but occasionally CT or MRI can be performed in cases of atypical presentation to exclude an ameloblastoma or a traumatic bone cyst [2]. No treatment is recommended; however, a follow-up examination at 36 months can be performed to ascertain lesion stability [3].

\section{Malignant lesions mimicking benign disease}

\section{Intraosseous mucoepidermoid carcinoma}

The vast majority of mucoepidermoid carcinomas arise within the major or minor salivary glands. Primary intraosseous mucoepidermoid carcinoma is an extremely rare tumour constituting less than $2 \%$ of all mucoepidermoid carcinomas. It arises centrally within the angle or posterior mandible [36]. Its aetiopathogenesis is not yet completely understood. Due to the paucity of cases reported in the literature, little is known regarding age of presentation and sex distribution. Clinical features include swelling and pain, as well as trismus. Imaging features are unilocular or multilocular, well-defined lesions with sclerotic borders, mimicking a benign cyst, a keratocyst or an ameloblastoma on OPT, CT and CBCT [37]. On MRI, the cystic tumour components display a low signal on T1, a high signal on T2 and no enhancement, whereas the nodular solid tumour components present a low signal on $\mathrm{T} 2$ and contrast
Fig. 16 Stafne cyst discovered incidentally. a OPT-like reconstruction from $\mathrm{CBCT}$ data set showing a well-defined, unilocular radiolucency (arrow) with sclerotic borders in the region of 37 and 38 . No relationship to teeth. b Axial, c coronal and $\mathbf{d}$ 3D reconstruction, posterior view. Bony defect (arrows) in characteristic location
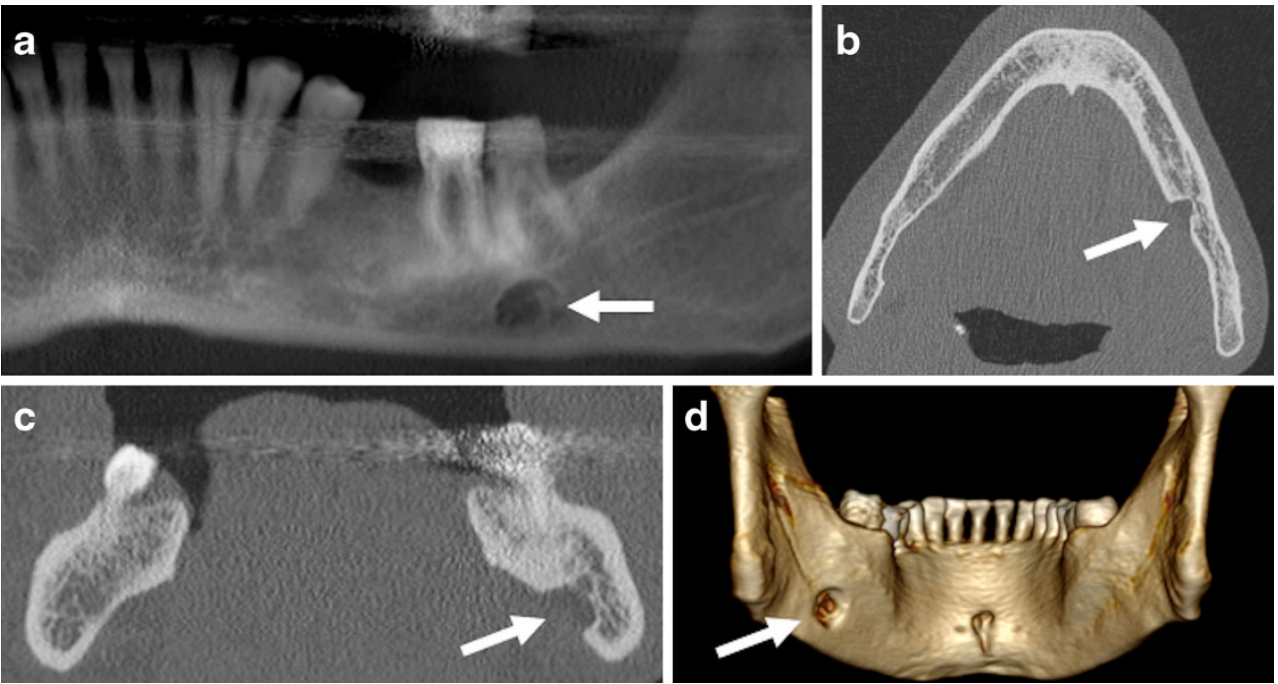

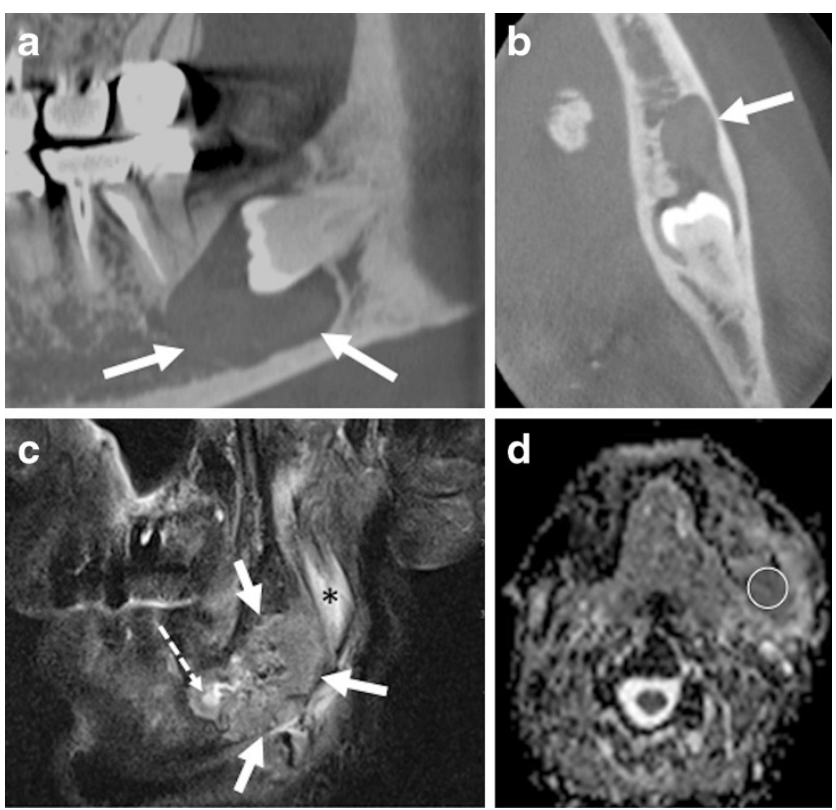

Fig. 17 Intracystic carcinoma. a Sagittal oblique and $\mathbf{b}$ axial CBCT image displaying the characteristic aspect of a dentigerous cyst with well-defined cyst borders (arrows). The cyst surrounds the crown of an impacted tooth. c T2-weighted image: solid intracystic component with moderately high signal intensity (arrows). Fluid with high signal intensity within the cyst (dashed arrow). Oedema in the masseter muscle (asterisk). d ADC map with low $\mathrm{ADC}$ values (circle) of solid parts $\left(\mathrm{ADC}=0.89 \times 10^{-3} \mathrm{~mm}^{2} / \mathrm{s}\right)$ suggesting an intracystic malignant tumour despite the benign appearance on CBCT. Histology revealed SCC arising within a dentigerous cyst

material enhancement. Extension into the muscles of mastication is common, as well as loco-regional lymph node metastases. MRI essential to narrow the differential diagnosis, as OPT and $\mathrm{CT}$ are non-specific. The presence of a destructive, infiltrative pattern with enhancing nodules within the cyst wall, as well as the presence of metastatic lymph nodes strongly suggests a malignant tumour, thus making biopsy mandatory.

\section{Primary intraosseous SCC}

Primary intraosseous SCC is a very rare neoplasm resulting from the malignant transformation of the epithelial lining of a residual cyst, follicular cyst or a keratocyst $[13,38]$. In consequence, as a general rule, following enucleation of any cystic lesion in the mandible, a thorough examination of the entire surgical specimen is mandatory to exclude the possibility of an intramural SCC that may have been overlooked preoperatively $[39,40]$. Primary intraosseous SCC has a predilection for men and typically occurs in the 6th-8th decade. There are less than 120 cases described in the literature. These tumours are aggressive lesions with a high metastatic potential. Due to the non-specific clinical findings, most intraosseous SCCs tend to be diagnosed at later stages. Panoramic radiographs, $\mathrm{CT}$ and $\mathrm{CBCT}$ findings mimic benign follicular cysts (Fig. 17) or radicular cysts. MRI and PET/CT are the only modalities suggesting a malignant tumour. Imaging findings on MRI are similar to those of intraosseous mucoepidermoid carcinoma: solid tumour portions have lower signal on $\mathrm{T} 2$, whereas cystic portions display a high signal. Enhancement of solid portions is typically seen after injection of contrast material, as well as infiltration into the perimandibular soft-tissues. ADC values of solid parts are low, suggesting tumour components with high cellularity (Fig. 17). On PET/CT, a high FDG uptake may be seen similar to SCC arising from the mucosa of the upper aero-digestive
Fig. 18 Mandibular NHL. a OPT. Difficulty to accurately identify the lesion due its median and paramedian position and the typical anterior OPT artefact (arrowheads) masking the lesion. Subtle enlargement of the right mental foramen (long arrow) compared with the normal contralateral side (short arrow). b, c T1-weighted unenhanced axial images. d T1-weighted fat-saturated contrast-enhanced sagittal image. Large infiltrative lesion (asterisks) extending from the vestibule through the mandible into the floor of the mouth (arrows). The mandibular cortical rim is mostly preserved although the lesion shows diffuse marrow infiltration
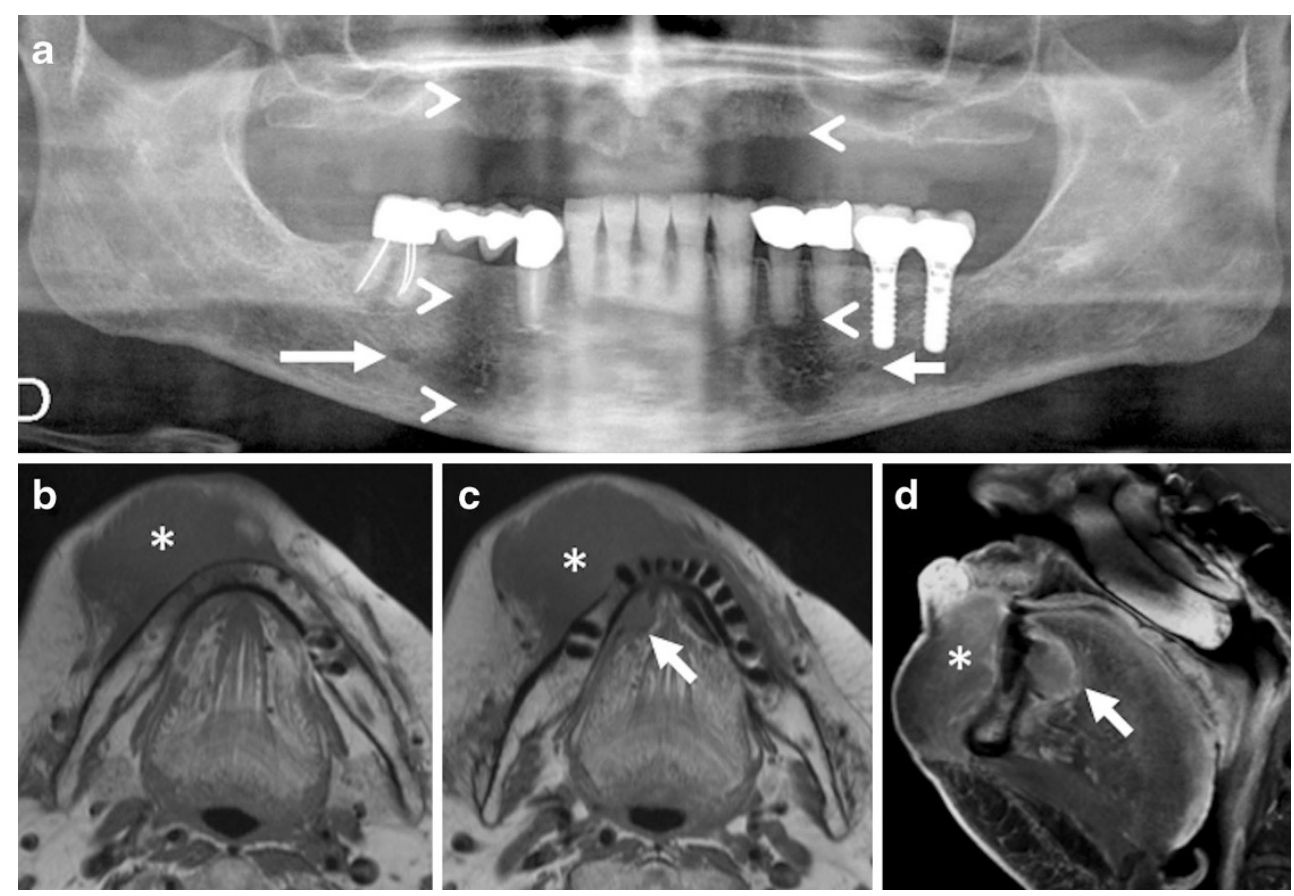
tract. Treatment consists of surgery with and without additional radiotherapy.

Malignant lesions that may be missed on OPT

\section{Mandibular lymphoma}

Lymphomas are malignant tumours developed from cells of the lymphatic system and can therefore affect any organ containing lymphoid tissue. While Hodgkin lymphomas (HL) most often involve lymph nodes in the head and neck, non-Hodgkin lymphomas (NHL) can equally affect extranodal sites [41] such as the lacrimal glands, salivary glands, muscles and soft tissues of the orbit, thyroid gland, maxilla and mandible. Mandibular NHL is very rare. As it is frequently mistaken for dental infection, a prolonged delay in diagnosis can occur. All age groups can be affected, however adults tend to be affected more often than children. There is no gender predilection. The clinical features of mandibular NHL are not specific and include pain, jaw swelling, ulceration, tooth mobility and cervical lymphadenopathy. On panoramic views, mandibular NHLs appear as ill-defined radiolucent lesions. Despite their large size, the tumours can be easily missed on conventional X-rays (Fig. 18). Radiological findings can be quite subtle such as teeth displacement in the occlusal direction and loss of the lamina dura with widening of the periodontal ligament space [41]. Rarely, mandibular NHL can present with minor osteolysis or with unilateral enlargement of the mandibular canal and mental foramen (Fig. 18). Enlargement of the mandibular canal and mental foramen is rare and has been reported in neurogenic tumours affecting the inferior alveolar nerve (schwannoma and neurofibroma), in perineural spread of disease (mainly from SCC, adenoid cystic carcinoma and lymphoma) and as an anatomic variant mimicking pathology [42]. In our experience, in mandibular NHL, CT and MRI typically show minor or no destruction of the cortical bone despite extensive infiltration of the bony marrow and of the perimandibular soft tissues (Fig. 18). As suggested in the literature, MRI provides superior visualisation of submucosal tumour extension and improved assessment of marrow infiltration compared with CT or CBCT [43]. Low signal on $\mathrm{T} 1$ and $\mathrm{T} 2$, homogenous contrast enhancement after injection of gadolinium chelates, absent areas of necrosis despite the large size (Fig. 18) and low ADC values (typically $0.6-0.8 \times 10^{-3} \mathrm{~mm}^{2} / \mathrm{s}$ ) are characteristic findings. On PET/CT, mandibular NHL display high FDG uptake and high SUV values (usually $\geq 10-15$ ), similar to lymphomas in other locations in the body. The differential diagnosis of mandibular NHL includes other infiltrative processes such as myeloma, leukaemia and bone metastases, making biopsy mandatory for the correct histological diagnosis. Treatment in mandibular NHL is done with chemo(radio)therapy.

\section{Conclusions}

The vast majority of radiolucent lesions of the mandible seen on conventional radiographs represent benign lesions that require no further work-up. Nevertheless, certain radiological features, such as large lesion size, bone scalloping, relationship to an impacted tooth or the mandibular canal, tooth resorption, as well as ill-defined lesion borders, require further radiological work-up. CT, CBCT, MRI and PET/CT are of additional help if the nature of the lesion is unclear and for the identification of those lesions, where biopsy is indicated for definitive histology.

Open Access This article is distributed under the terms of the Creative Commons Attribution License which permits any use, distribution, and reproduction in any medium, provided the original author(s) and the source are credited.

\section{References}

1. Dunfee BL, Sakai O, Pistey R, Gohel A (2006) Radiologic and pathologic characteristics of benign and malignant lesions of the mandible. Radiographics 26:1751-1768

2. Devenney-Cakir B, Subramaniam RM, Reddy SM, Imsande H, Gohel A, Sakai O (2011) Cystic and cystic-appearing lesions of the mandible: review. AJR Am J Roentgenol 196:WS66-WS77. doi:10. 2214/AJR.09.7216

3. Scholl RJ, Kellett HM, Neumann DP, Lurie AG (1999) Cysts and cystic lesions of the mandible: clinical and radiologic-histopathologic review. Radiographics 19:1107-1124

4. Cotti E, Campisi G (2004) Advanced radiographic techniques for the detection of lesions in bone. Endod Top 7:52-72

5. Minami M, Kaneda T, Ozawa K, Yamamoto H, Itai Y, Ozawa M et al (1996) Cystic lesions of the maxillomandibular region: MR imaging distinction of odontogenic keratocysts and ameloblastomas from other cysts. AJR Am J Roentgenol 166:943-949

6. Schuknecht BF, Carls FR, Valavanis A, Sailer HF (1997) Mandibular osteomyelitis: evaluation and staging in 18 patients, using magnetic resonance imaging, computed tomography and conventional radiographs. J Craniomaxillofac Surg 25:24-33

7. Varoquaux A, Rager O, Lovblad KO, Masterson K, Dulguerov P, Ratib O et al (2013) Functional imaging of head and neck squamous cell carcinoma with diffusion-weighted MRI and FDG PET/CT: quantitative analysis of ADC and SUV. Eur J Nucl Med Mol Imaging 40:842-852

8. Varoquaux A, Rager O, Poncet A, Delattre BM, Ratib O, Becker CD et al (2013) Detection and quantification of focal uptake in head and neck tumours: F-FDG PET/MR versus PET/CT. Eur J Nucl Med Mol Imaging. doi:10.1007/s00259-013-2580-y

9. Freitas DQ, Tempest LM, Sicoli E, Lopes-Neto FC (2006) Bilateral dentigerous cysts: review of the literature and report of an unusual case. Dentomaxillofac Radiol 35:464-468

10. Maeda T, Tateishi U, Terauchi T et al (2007) Unsuspected bone and soft tissue lesions identified at cancer screening using positron emission tomography. Jpn J Clin Oncol 37:207-215

11. Barnes L, Eveson JW, Reichart P, Sidransky D (2005) World Health Organization Classification of Tumours. Pathology and genetics of head and neck tumours. IARC Press, Lyon 
12. Sham E, Leong J, Maher R, Schenberg M, Leung M, Mansour AK (2009) Mandibular ameloblastoma: clinical experience and literature review. ANZ J Surg 79:739-744

13. Colbert S, Brennan PA, Theaker J, Evans B (2012) Squamous cell carcinoma arising in dentigerous cysts. J Craniomaxillofac Surg 40: e355-e357

14. Ali M, Baughman RA (2003) Maxillary odontogenic keratocyst. J Am Dent Assoc 134:877-883

15. Shear M (2003) Odontogenic keratocysts: natural history and immunohistochemistry. Oral Maxillofac Surg Clin N Am 15:347-362

16. Lindeboom JA, Kroon FH, de Vires J, van den Akker HP (2003) Multiple recurrent and de novo odontogenic keratocysts associated with oral-facial-digital syndrome. Oral Surg Oral Med Oral Pathol Oral Radiol Endod 95:458-462

17. Nguyen BD (2005) Malignant ameloblastoma with thoracic vertebral metastasis: PET/CT and MR imaging. Clin Nucl Med 30:450-452

18. Sabino-Bezerra JR et al (2012) Atypical presentations of simple bone cysts of the mandible: a case series and review of literature. $\mathrm{J}$ Craniomaxillofac Surg 41:391-396. doi:10.1016/j.jcms.2012.11.002

19. Lee BD, Lee W, Lee J, Son HJ (2013) Eosinophilic granuloma in the anterior mandible mimicking radicular cyst. Imaging Sci Dent 43: $117-122$

20. Mueller WP, Melzer HI, Schmid I, Coppenrath E, Bartenstein P, Pfluger T (2013) The diagnostic value of 18F-FDG PET and MRI in paediatric histiocytosis. Eur J Nucl Med Mol Imaging 40:356-363

21. Schutz P, El-Bassuoni KH, Munish J, Hamed HH, Padwa BL (2010) Aggressive central giant cell granuloma of the mandible. J Oral Maxillofac Surg 68:2537-2544

22. Gupta M, Singh S, Kaur R (2013) Central giant cell granuloma of the maxilla. BMJ Case Rep. doi:10.1136/bcr-2013-009102

23. Van Schuppen J, van Doorn MM, van Rijn RR (2012) Childhood osteomyelitis: imaging characteristics. Insights Imaging 3:519-533

24. Baltensperger M, Eyrich GK (2008) Definition and classification. In: Baltensperger M, Eyrich GK (eds) Osteomyelitis of the jaws. Springer, Berlin Heidelberg, pp 5-50

25. Pincus DJ, Armstrong MB, Thaller SR (2009) Osteomyelitis of the craniofacial skeleton. Semin Plast Surg 23:73-79. doi:10.1055/s0029-1214159

26. Advisory Task Force on Bisphosphonate-Related Ostenonecrosis of the Jaws, American Association of Oral and Maxillofacial Surgeons (2007) American Association of Oral and Maxillofacial Surgeons position paper on bisphosphonate-related osteonecrosis of the jaws. J Oral Maxillofac Surg 65:369-376

27. Bisdas S, Chambron Pinho N, Smolarz A, Sader R, Vogl TJ, Mack MG (2008) Biphosphonate-induced osteonecrosis of the jaws: CT and MRI spectrum of findings in 32 patients. Clin Radiol 63:71-77

28. Becker M, Schroth G, Zbären P, Delavelle J, Allal A, Greiner R, Rüfenacht DA, Vock P, Terrier F (1997) Long-term changes induced by high-dose irradiation of the head and neck region: imaging findings. Radiographics 17:5-26
29. Chong J, Hinckley LK, Ginsberg LE (2000) Masticator space abnormalities associated with mandibular osteoradionecrosis: MR and CT findings in five patients. AJNR Am J Neuroradiol 21:175-178

30. Lambade PN, Lambade D, Goel M (2013) Osteoradionecrosis of the mandible: a review. Oral Maxillofac Surg 17:243-249. doi:10.1007/ s10006-012-0363-4

31. Kaur H, Verma S, Jawanda MK, Sharma A (2012) Aggressive osteoblastoma of the mandible: a diagnostic dilemma. Dent Res J (Isfahan) 9:334-337

32. Jimi E, Furuta H, Matsuo K, Tominaga K, Takahashi T, Nakanishi O (2010) The cellular and molecular mechanisms of bone invasion by oral squamous cell carcinoma. Oral Dis 17:462-468. doi:10.1111/j. 1601-0825.2010.01781.x

33. Trotta BM, Pease CS, Rasamny JJ, Raghavan P, Mukherjee S (2011) Oral cavity and oropharyngeal squamous cell cancer: key imaging findings for staging and treatment planning. Radiographics 31:339354

34. Barnes L (2009) Metastases of the head and neck: an overview. Head Neck Pathol 3:217-224

35. Scolozzi P, Becker M, Lombardi T (2012) Mandibular condylar metastasis mimicking acute internal derangement of the temporomandibular joint. J Can Dent Assoc 78:c77

36. Raut D, Khedkar S (2009) Primary intraosseous mucoepidermoid carcinoma of the maxilla: a case report and review of literature. Dentomaxillofac Radiol 38:163-168

37. Martínez-Madrigal F, Pineda-Daboin K, Casiraghi O, Luna MA (2000) Salivary gland tumors of the mandible. Ann Diagn Pathol 4: 347-353

38. Bodner L, Manor E, Shear M, van der Waal I (2011) Primary intraosseous squamous cell carcinoma arising in an odontogenic cyst: a clinicopathologic analysis of 116 reported cases. J Oral Pathol Med 40:733-738

39. Nomura T, Monobe H, Tamaruya N, Kishishita S, Saito K, Miyamoto R, Nakao K (2013) Primary intraosseous squamous cell carcinoma of the jaw: two new cases and review of the literature. Eur Arch Otorhinolaryngol 270:375-379. doi:10.1007/s00405-012-2235-9

40. Manor E, Kachko L, Puterman MB, Szabo G, Bodner L (2012) Cystic lesions of the jaws - a clinicopathological study of 322 cases and review of the literature. Int J Med Sci 9:20-26

41. Gonzalez-Fontal GR, Rosales JD, Jaramillo R, Henao-Martinez AF (2011) Primary extranodal, extralymphatic Hodgkin lymphoma of the mandible. Case Rep Med 2011:387570. doi:10.1155/2011/ 387570

42. Terzic A, Becker M, Imholz B, Scolozzi P (2013) Unilateral widening of the inferior alveolar nerve canal: a rare anatomic variant mimicking disease. Oral Radiol 29:160-165

43. Imaizumi A, Kuribayashi A, Watanabe H, Ohbayashi N, Nakamura S, Sumi Y et al (2012) Non-Hodgkin lymphoma involving the mandible: imaging findings. Oral Surg Oral Med Oral Pathol Oral Radiol 113:e33-e39 